\title{
Superrotation and Nonlinear Hadley Circulation Response to Zonally Asymmetric Sea Surface Temperature in an Aquaplanet GCM
}

\author{
Masato MORI, Masahiro WATANABE, and Masahide KIMOTO \\ Atmosphere and Ocean Research Institute, The University of Tokyo, Kashiwa, Japan \\ (Manuscript received 21 June 2010, in final form 19 September 2012)
}

\begin{abstract}
The responses of the equatorial zonal wind and the Hadley circulation to the equatorial zonal wavenumber one sea surface temperature (SST) anomaly, $T_{s}^{*}$, are examined in an atmospheric general circulation model (AGCM) with an aquaplanet condition. The Hadley cell is weakened as the magnitude of $T_{s}^{*}$ increases, balancing with a decrease in the zonal-mean diabatic heating over the tropics. The decrease of heating reflects a nonlinear relationship between precipitation and SST; deep convection, such as a super cloud cluster, is significantly suppressed over cold $T_{s}{ }^{*}$, whereas is slightly enhanced over warm $T_{s}^{*}$. The effective suppression of deep convection is accomplished by the stable boundary layer and the dry subsidence anomaly associated with the Walker cell which is excited by the SST anomaly. And the decreased convection acts to further reinforce the subsidence via thermodynamic balance. Therefore, this positive feedback between large-scale circulation and deep convection determines the nonlinear relationship and controls the strength of the Hadley cell.

In terms of the energetics of the tropical circulation, the Hadley cell has to be weakened to compensate for the lack of energy supply caused by an increase of tropical radiative cooling due to the effective suppression of deep convection over cold $T_{s}^{*}$.

We compared the results of our AGCM with that of other 15 aquaplanet AGCMs integrated with the same SST distribution. While the Hadley cell is weakened in all AGCMs when $T_{s}^{*}$ is added to the zonal uniform SST, there is a large diversity in the strength. This suggests that the difference in the physical parameterization causes a different sensitivity of the Hadley cell response to zonally asymmetric SST. The magnitude of weakening is approximately proportional to the decreased (increased) amount of the deep convective precipitation (the radiative cooling) over the tropics. This strong relationship suggests that the positive feedback also works in other AGCMs. It is considered that the feedback is also important for understanding the formation of a real tropical climate.
\end{abstract}

Keywords Hadley circulation; Walker circulation; superrotation; super cloud cluster; nonlinear response of precipitation to SST

\section{Introduction}

The question of how the tropical climatological circulation, such as the Hadley and Walker cell, is regulated by sea surface temperature (SST) has been a major topic in the meteorological literature. For example, several theoretical and numerical studies

Corresponding author: Masato Mori, Atmosphere and Ocean Research Institute, The University of Tokyo 5-1-5 Kashiwanoha, Kashiwa, Chiba 277-8568, Japan

E-mail: masato@aori.u-tokyo.ac.jp

C2013, Meteorological Society of Japan have been conducted to explain how a latitudinal curvature and strength of the equatorial SST control the strength and width of the Hadley cell (e.g., Held and Hou 1980).

Using a global dry two-level primitive equation model, Suarez and Duffy (1992) and Saravanan (1993) demonstrated that a tropical climate having an upperlevel easterly abruptly switches to an equatorial superrotating state that accompanies a zonal-mean westerly and weak Hadley cell. This regime change in the zonalmean circulation occurs when a strong zonal wavenumber two tropical eddy heating is superimposed on 

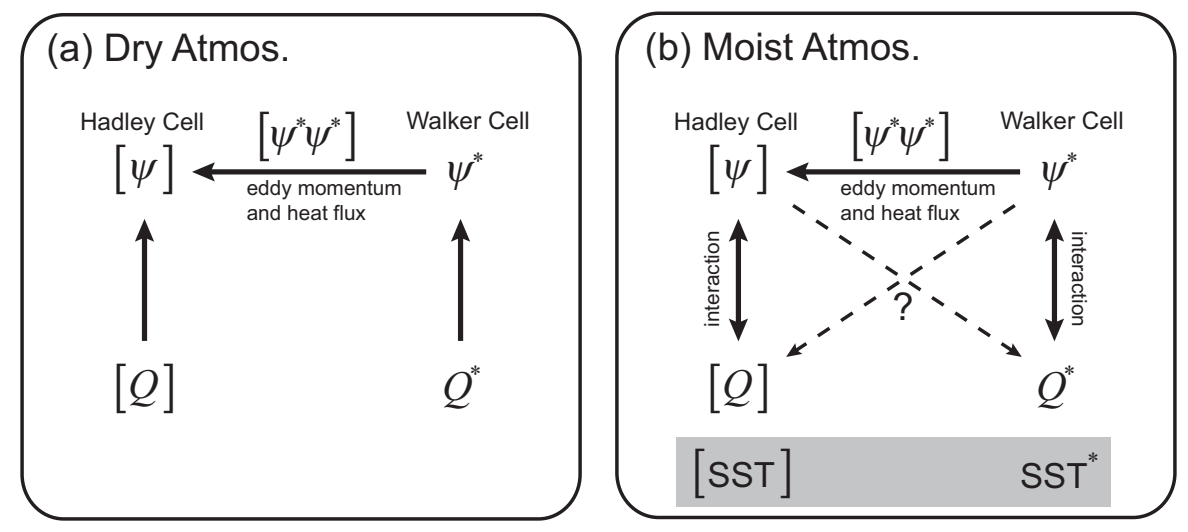

Fig. 1. Schematic diagram of (a) dry and (b) moist atmospheres. For a meteorological field $\psi$, $[\psi]$ and $\psi^{*}$ denote zonal mean and deviation from the zonal mean, respectively.

a zonally uniform heating. Upper tropospheric superrotation and the weakening of the Hadley cell have also been found in multi-level atmospheric general circulation models (AGCMs) with idealized physics and imposed zonally asymmetric tropical heating (Kraucunas and Hartmann 2005, hereafter KH05) and in aquaplanet AGCMs with zonally asymmetric SST over the equator (Battisti and Ovens 1995; Hoskins et al. 1999; Inatsu et al. 2002). However, the sudden transition to superrotation has not been found in such AGCMs (Hoskins et al. 1999; KH05).

Hide's theorem (Hide 1969; Held and Hou 1980) demands that the upper tropospheric superrotating state be maintained by eddy angular momentum fluxes that are directed up the local angular momentum gradient (KH05). In two-level models, it is pointed out that the momentum provided by midlatitude transient eddies is required to maintain the superrotation (Suarez and Duffy 1992; Saravanan 1993). In contrast, Hoskins et al. (1999) and KH05 demonstrated that the superrotating state in multi-level AGCMs was maintained by the eddy momentum flux associated with the stationary waves induced by the imposed equatorial thermal contrast (Matsuno 1966; Gill 1980). Some arguments suggest that the interaction between transient eddies and mean flow cannot be actually simulated with only two vertical levels (see KH05 for detailed arguments). However, it remains unclear why the Hadley cell is weakened when forced by a zonally asymmetric thermal forcing or SST.

The tropical climate may be determined by complex interactions between the large-scale atmospheric circulation, SST, and physical processes such as radiative transfer, clouds, and convection. In a dry atmosphere, however, the dynamics of the Hadley cell will likely be much simpler (Fig. 1a): The Hadley cell (denoted as $[\psi]$ ) is controlled by the zonal-mean component of equatorial heating $([Q])$ and convergence of the eddy momentum and heat fluxes $\left(\left[\psi^{*} \psi^{*}\right]\right)$ induced by the zonally asymmetric component of heating $\left(Q^{*}\right)$. The control variable of the equatorial heat source, $Q^{*}$, is imposed and fixed in time (Suarez and Duffy 1992; Saravanan 1993; KH05).

In contrast, in a moist atmosphere (Fig. 1b), the control variable will be SST (denoted as $T_{S}$ in this paper). It will change an equatorial heating via precipitation process, whereas the heating will interact with circulation. Then, how does the zonally asymmetric equatorial SST $\left(T_{s}^{*}\right)$ regulate tropical large-scale circulation, such as the Hadley and Walker cells? This study aims to address this question and clarify the interaction between tropical rainfall and circulation that controls the Hadley cell. This issue has not been fully examined in previous studies using moist AGCMs (Battisti and Ovens 1995; Hoskins et al. 1999; Inatsu et al. 2002). To answer the above question, in this study, a series of experiments are conducted using a full AGCM with aquaplanet condition. In these AGCM experiments, we keep the zonal-mean SST ([T $\left.\left.T_{s}\right]\right)$ constant and change only $T_{S}^{*}$ to examine the mechanism by which the zonally asymmetric SST regulates the Hadley circulation via changing the Walker circulation. It should be noted that, even though the zonal-mean SST does not change, zonal-mean heating may not be the same (Fig. 1b).

Recently, a project for intercomparison of idealized climates simulated by different AGCMs with controlled aquaplanet conditions was proposed (Aqua- 
Planet Experiment Project: APE; Neale and Hoskins 2001ab; Blackburn et al. 2013; Williamson et al. 2013; Williamson et al. 2012). This project aims to provide a benchmark of current model behavior and understand the causes of inter-model differences. For these purposes, each AGCM is integrated with the same set of several idealized distributions of SST. To estimate robustness and understand the dependence of our experimental results on subgrid-scale parameterization, outputs of several AGCMs are also analyzed in this study.

This paper is organized as follows: In Section 2, we present the model and experimental design. In Section 3 , we first examine equatorial superrotation. The weakening mechanisms of the Hadley cell are investigated in Section 4, with emphasis on the interaction between large-scale circulation and convection. In Section 5, models are compared in terms of superrotation and Hadley cell responses. Section 6 provides summary and discussion.

\section{Model and experimental design}

The AGCM used is the atmospheric component of a coupled global climate model called the Model for Interdisciplinary Research on Climate (MIROC) (Hasumi and Emori 2004), which is a Japanese community model collaboratively developed at the Center for Climate System Research (CCSR), University of Tokyo, the National Institute for Environmental Studies (NIES), and the Frontier Research Center for Global Change (FRCGC). MIROC has been contributing to the Intergovernmental Panel on Climate Change (IPCC) Fourth Assessment Report (e.g., Lin et al. 2006) and is widely used to study past, present, and future climate change (Kimoto 2005; Nozawa et al. 2005; Watanabe 2008; Mochizuki et al. 2010; among others). The atmospheric component model, referred to as the CCSR/NIES/FRCGC AGCM, contains the standard physics package such as cumulus convection, cloud water, turbulence closure, and radiative transfer schemes. The cumulus parameterization scheme is based on Arakawa and Schubert (1974), and the details are updated by a prognostic closure based on Pan and Randall (1998).

The model resolution employed is horizonal T42 and $20 \sigma$-vertical levels. The model is run following APE framework proposed by Neale and Hoskins (2001a, b). Among eight perpetual-equinox experiments, each of which is driven by a different SST distribution, we concentrate on the "control" (denoted as CTL) and "3kw1" experiments that are forced by the SST, defined as

$$
\begin{aligned}
T_{s}(\lambda, \varphi) & = \begin{cases}273.15+T_{0}\left[1-\sin ^{2}\left(\frac{90}{60} \varphi\right)\right], & \text { for }|\varphi|<\varphi_{d} \\
273.15, & \text { otherwise }\end{cases} \\
T_{s}^{*}(\lambda, \varphi) & = \begin{cases}\alpha \cos \left(\lambda-\lambda_{0}\right) \cos ^{2}\left(\frac{90}{30}|\varphi|\right), & \text { for }|\varphi|<\varphi_{d} / 2, \\
0, & \text { otherwise }\end{cases}
\end{aligned}
$$

where $T_{S}$ is given to CTL and $T_{s}^{*}$ is the SST anomaly with the zero zonalmean; $T_{s}+T_{s}^{*}$ is given to $3 \mathrm{kw} 1$. Other parameters are $T_{0}=27 \mathrm{~K}, \alpha=3 \mathrm{~K}, \lambda_{0}=0^{\circ}$, and $\varphi_{d}=60^{\circ}$. Figure 2 shows the distribution of $T_{s}$ and $T_{s}{ }^{*}$. It is apparent that CTL is driven by a zonally uniform SST with a maximum of $27^{\circ} \mathrm{C}$ along the equator while $3 \mathrm{kw} 1$ is forced by a zonal wavenumber one patch of tropical warm and cold pools between $30^{\circ} \mathrm{S}$ and $30^{\circ} \mathrm{N}$, superimposed on the zonally uniform SST. In addition to the experiments following APE regulation, we conducted additional experiments in which the zonally uniform SST remains the same but the amplitude parameter $\alpha$ is varied in the range of $1 \leq \alpha \leq 9$ with an interval of $1 \mathrm{~K}$; these experiments are denoted as $1 \mathrm{kw} 1$, $2 \mathrm{kw} 1, \ldots, 9 \mathrm{kw} 1$ (Fig. 2c). After six-month spinup, the model was integrated for three years for each run. The statistics from the last three years are used to characterize the mean climate in each experiment.

It is useful to know which experimental configuration is the prototype for observations. Figure $3 \mathrm{c}$ shows the long-term averaged SST at the equator derived from the Met Office Hadley Centre's sea ice and sea surface temperature data set (HadISST; Rayner et al. 2003). Although the observational SST contains waves with various zonal wavenumbers, the zonal wavenumber zero (corresponding to the zonal-mean of $27^{\circ} \mathrm{C}$ ) and one components are distinguished and are similar to the SST of $3 \mathrm{kw} 1$ (Fig. 3f). Furthermore, the spatial distribution of the eddy (i.e., deviation from the zonal-mean) component of the long-term averaged streamfunction $\left(\bar{\psi}^{*}\right)$ in the upper and lower troposphere (Figs. 3a, b), derived from European Centre for Medium-Range Weather Forecasts 40-year reanalysis (ERA40; Uppala et al. 2005), is also similar to $\bar{\psi}^{*}$ in $3 \mathrm{kw} 1$ (Figs. 3d, e). Therefore, as far as our experiments are concerned, $3 \mathrm{kw} 1$ is the most realistic configuration.

\section{Superrotation}

Before examining the Hadley cell responses, it is useful to investigate the equatorial superrotation and confirm the consistency with previous studies. The time- and zonal-mean zonal wind $(\overline{[u]})$ in the CTL case is shown in Fig. 4a. Because asymmetries of land/ sea distribution or orography are absent, the zonal wind is latitudinally narrow and is considerably stronger than observation. However, it closely resembles the 
(a)

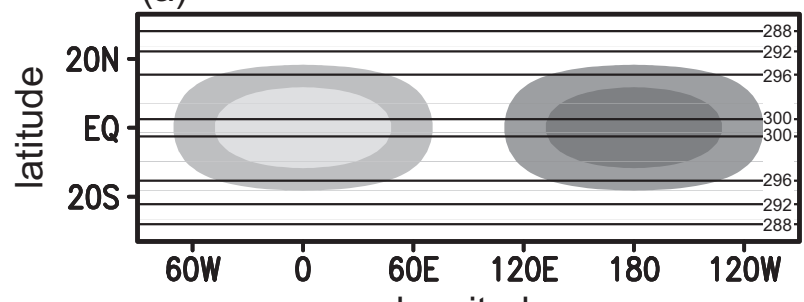

(c)

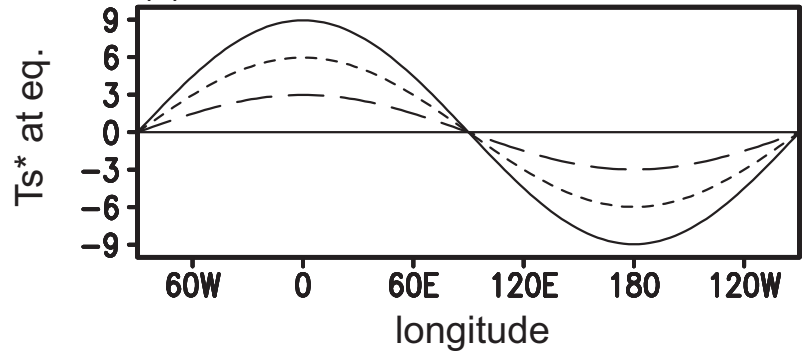

(b)

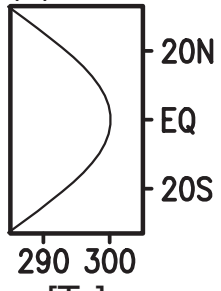

[Ts]

Fig. 2. (a) Spatial structure of $T_{S}$ (contour) and $T_{S}{ }^{*}$ in $3 \mathrm{kw} 1$ (shade). The contour interval is $4 \mathrm{~K}$. (b) Profile of zonally averaged $T_{s}$. (c) Cross section of $T_{S}{ }^{*}$ at the equator. Dash, dotted, and solid curves denote $T_{S}{ }^{*}$ with $\alpha$ values of 3,6 , and 9 , respectively.

result simulated in another AGCM with identical SST distribution (Neal and Hoskins 2001b), except that the upper tropospheric zonal wind is weak westerly over the equator. Therefore, in our model, very weak superrotation occurs in the absence of the zonal asymmetry in SST; this is different from the results in dry multi-level (KH05) and two-level AGCMs (Suarez and Duffy 1992; Saravanan 1993) and in other aquaplanet AGCMs (e.g., Battisti and Ovens 1995; Hoskins et al. 1999; Neal and Hoskins 2001b). However, our results are similar to those of Lee (1999) and Nasuno (2008), who showed a weak westerly response to a zonally uniform $\mathrm{SST}^{1}$.

In $3 \mathrm{kw} 1$ and 9kw1 experiments (Figs. 4b, c), the upper tropospheric westerly over the equator is strengthened and extended into mid-troposphere as $\alpha$ increases. The sudden transition to the superrotating state shown in two-level models (Suarez and Duffy 1992; Saravanan 1993) is not seen in our AGCM, but the westerly is gradually becoming stronger as pointed out in the previous studies (Hoskins et al. 1999; KH05).

To identify relative roles of the different dynamical mechanisms that have been proposed for the formation

\footnotetext{
${ }^{1}$ In Section 5, it will be shown that the difference in physical parameterizations yields significant inter-model differences in the polarity of $\overline{[u]}$ in CTL even though identical SST is used.
}

of superrotation, such as the momentum flux convergence associated with transient and stationary eddies, momentum budget is examined using the time and zonally averaged zonal momentum equation, which can be decomposed as

$$
\frac{\partial \overline{[u]}}{\partial t}=\sum_{i=1}^{7} \xi_{i},
$$

where

$$
\begin{aligned}
& \xi_{1}=\left(f-\frac{1}{a \cos \varphi} \frac{\partial}{\partial \varphi}(\overline{[u]} \cos \varphi)\right) \overline{[v]}, \\
& \xi_{2}=-\overline{[w]} \frac{\partial \overline{[u]}}{\partial p}, \\
& \xi_{3}=-\frac{1}{a \cos ^{2} \varphi} \frac{\partial}{\partial \varphi}\left(\left[\bar{u}^{*} \bar{v}^{*}\right] \cos ^{2} \varphi\right), \\
& \xi_{4}=-\frac{\partial}{\partial p}\left[\bar{u}^{*} \bar{w}^{*}\right], \\
& \xi_{5}=-\frac{1}{a \cos ^{2} \varphi} \frac{\partial}{\partial \varphi}\left(\overline{\left[u^{\prime} v^{\prime}\right]} \cos ^{2} \varphi\right), \\
& \xi_{6}=-\frac{\partial}{\partial p} \overline{\left[u^{\prime} w^{\prime}\right]}, \\
& \xi_{7}=\overline{\left[F_{x}\right]},
\end{aligned}
$$

$u$ is the zonal wind, $v$ the meridional wind, $w$ the vertical pressure velocity, $f$ the Coriolis parameter, $a$ 
(a) $\bar{\psi}^{*}$ at $300 \mathrm{hPa}$

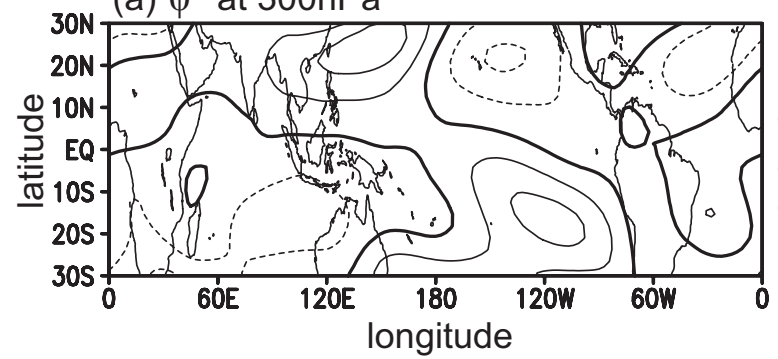

(b) $\bar{\psi}^{*}$ at $850 \mathrm{hPa}$

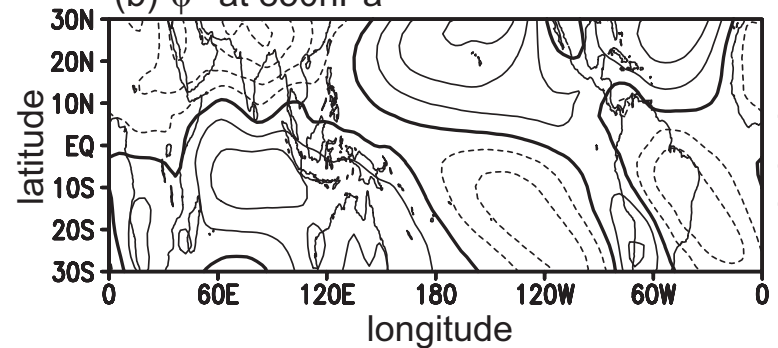

(c) SST at equator (HadISST)

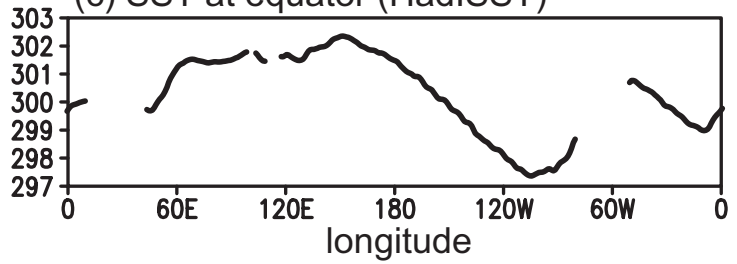

(d) $\bar{\psi}^{*}$ at $300 \mathrm{hPa}$ and $\mathrm{Ts}^{*}(3 \mathrm{kw} 1)$

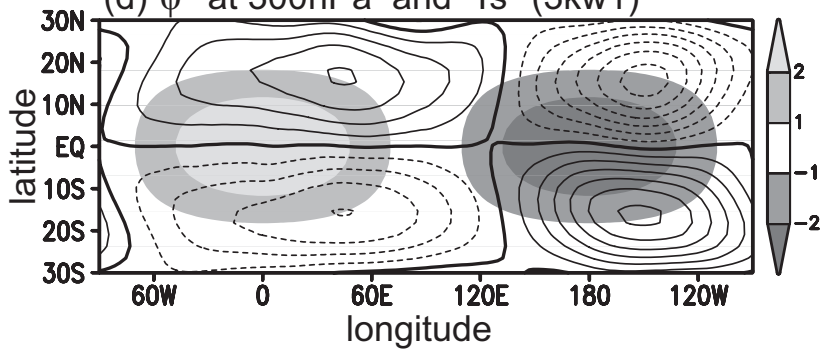

(e) $\bar{\psi}^{*}$ at $850 \mathrm{hPa}$ and $\mathrm{Ts}^{*}(3 \mathrm{kw} 1)$
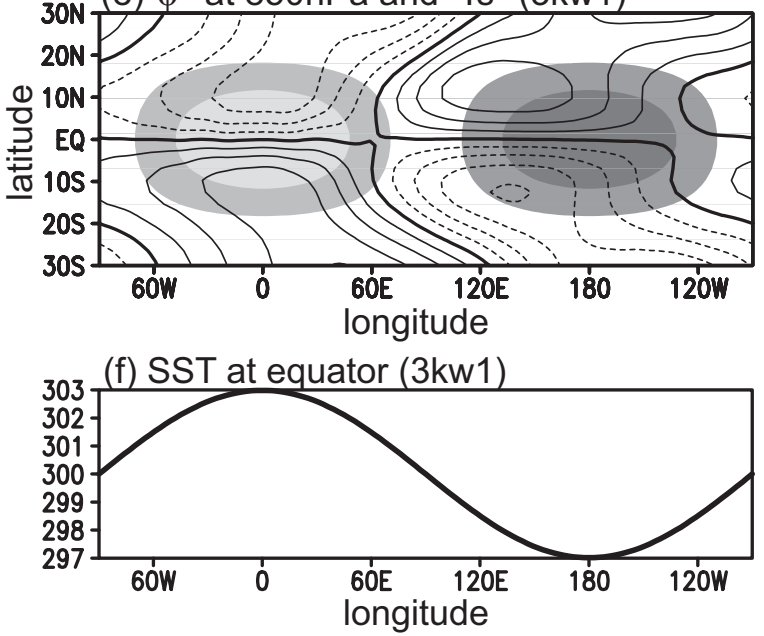

Fig. 3. Stationary eddy components of streamfunction $\bar{\psi}^{*}$ at (a) $300 \mathrm{hPa}$ (contour int. is $4 \times 10^{6} \mathrm{~m}^{2} \mathrm{~s}^{-1}$ ) and (b) $850 \mathrm{hPa}$ (contour int. is $2 \times 10^{6}$ ) derived from ERA40. (c) Cross section of the annual averaged SST (unit is K) at the equator derived from HadISST. All data of the period of coverage extends from 1957 to 2002 (from 1870 to 2004) and are used to calculate the time mean of streamfunction (SST). (d)-(e) Same as (a)-(b) but the aquaplanet GCM responses in $3 \mathrm{kw} 1 . T_{S}{ }^{*}$ in $3 \mathrm{kw} 1$ is superimposed (shade). (f) Cross section of imposed $\mathrm{SST}\left(T_{S}+T_{S}{ }^{*}\right)$ in $3 \mathrm{kw} 1$. The zero contour is thickened and negative contours are dashed.

the earth radius, and $F_{x}$ the frictional dissipation term. The overbars (brackets) and primes (asterisks) denote the time (zonal) mean and deviation from the time (zonal) mean, respectively.

It is important to explain the physical meaning of each term in Eq. (3). The term $\xi_{1}$ is the meridional advection of absolute vorticity by mean meridional circulation, $\xi_{2}$ the vertical advection of zonal momentum, and $\xi_{3}$ and $\xi_{4}\left(\xi_{5}\right.$ and $\left.\xi_{6}\right)$ the zonal accelerations associated with steady (transient) eddy momentum fluxes. Note that the first two terms represent the zonal accelerations associated with the Hadley circulation, while the third and fourth terms are related to the Walker circulation.

Figure 5 shows the equatorial zonal momentum budget at $200 \mathrm{hPa}$ as a function of the $T_{s}{ }^{*}$ magnitude $\alpha$. In CTL experiment, the total momentum budget (Fig. 5a) demonstrates that the transient eddy terms are dominant and are balanced by Hadley cell terms. In particular, the dominant balance is between $\xi_{5}$ and $\xi_{2}$ terms (Figs. 5b-d), which indicates that the weak westerly over the equatorial upper troposphere (Fig. $4 a)$ is maintained by the meridional convergence of momentum associated with the transients, and the Hadley cell acts to suppress it because of the local negative vertical shear of the mean zonal wind.

The above dynamical balance for $\overline{[u]}$ eventually changes as the zonally asymmetric SST is amplified. The zonal accelerations associated with the steady eddy terms are dominant as $\alpha$ increases, while the accelerations associated with the transients approach zero (Fig. 5a). These results indicate that the upper tropospheric steady wave response to $T_{S}^{*}$ is responsible for driving the superrotation under the zonally asymmetric SST boundary conditions, 
(a) zonal wind : CTL

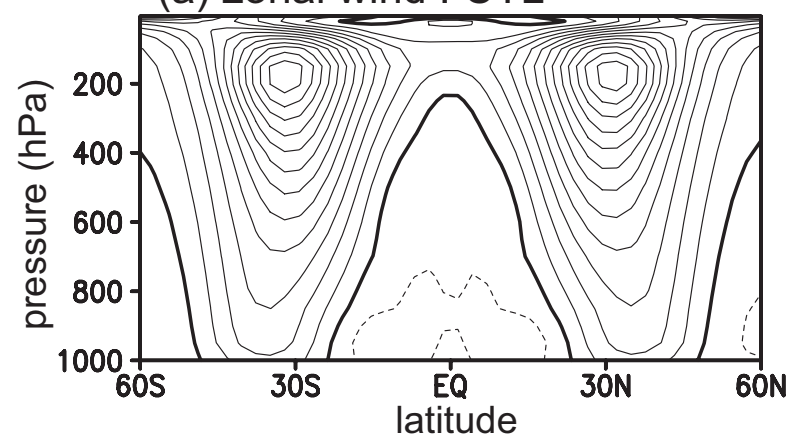

(b) zonal wind : $3 \mathrm{kw} 1$

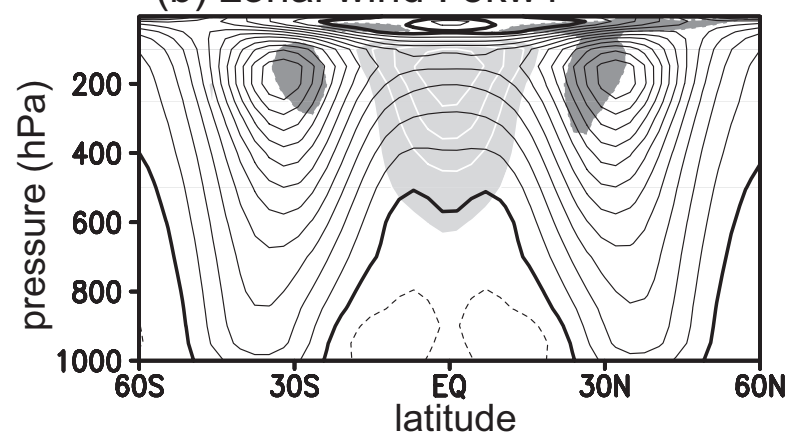

(c) zonal wind : $9 \mathrm{kw} 1$

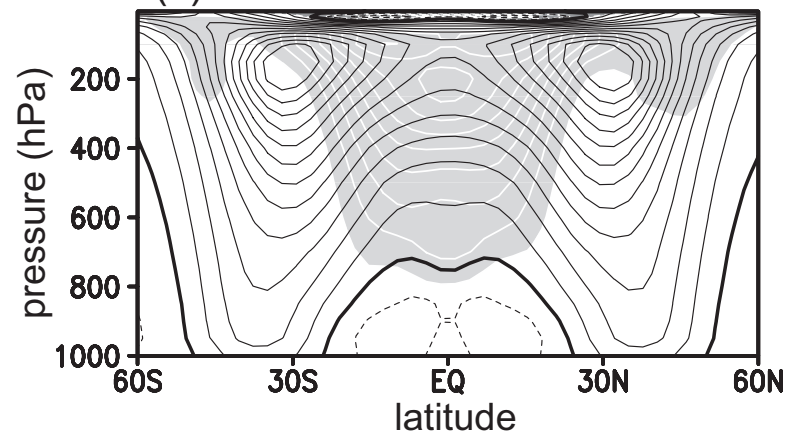

(d) streamfunction : CTL

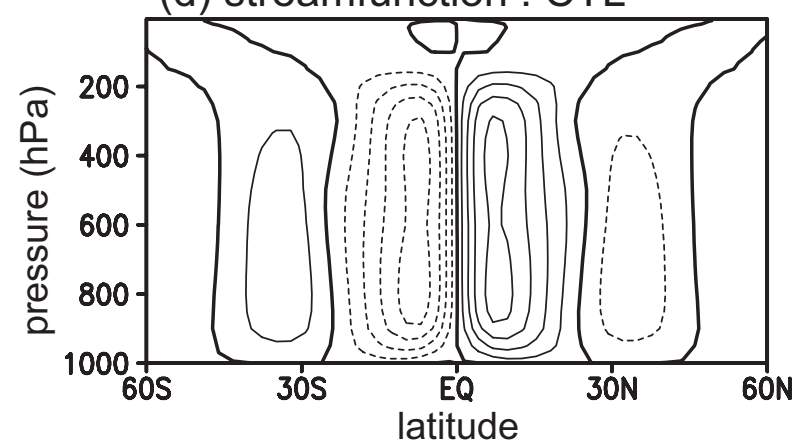

(e) streamfunction : 3kw1

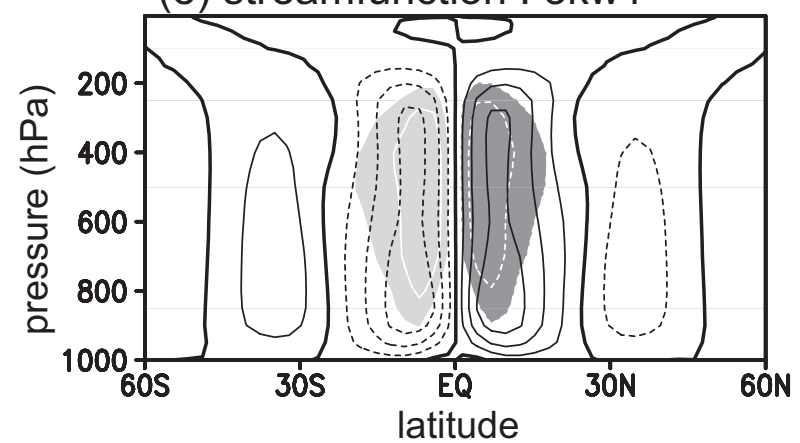

(f) streamfunction : 9kw1

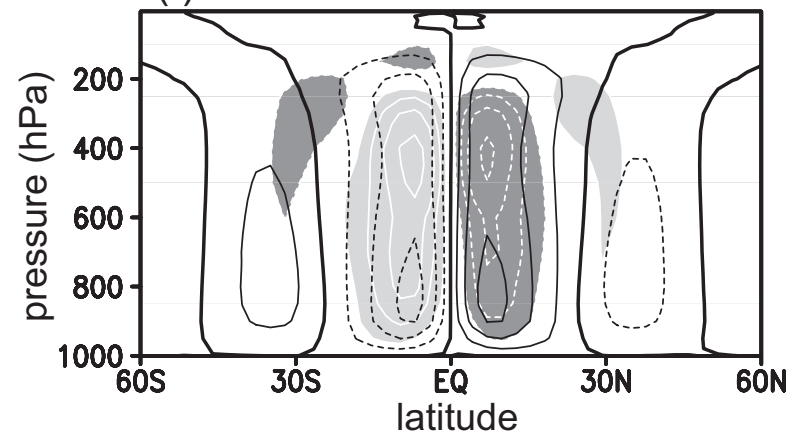

Fig. 4. Time and zonally averaged zonal winds $[\bar{u}]$ in: (a) CTL, (b) $3 \mathrm{kw} 1$, and (c) 9kw1. The contour interval is $5 \mathrm{~m} \mathrm{~s}^{-1}$. The deviation from CTL in which the value is larger (smaller) than $3(-3)$ is shaded as light (dark) gray in (b)-(c) and is denoted by white contours at intervals of three. Mean meridional streamfunction $\chi$ responses in: (d) CTL, (e) $3 \mathrm{kw} 1$, and (f) $9 \mathrm{kw} 1$. The contour interval is $1 \times 10^{4} \mathrm{~m} \mathrm{~s}^{-1} \mathrm{~Pa}$. The deviation from CTL in which the value is larger (smaller) than $4(-4) \times 10^{3}$ is shaded as light (dark) gray in (d)-(f) and is denoted by white contours at intervals of $4 \times 10^{3}$. The zero contour is thickened and negative contours are dashed.

which is consistent with the results of previous studies (Hoskins et al. 1999; KH05). Although the steady eddy terms are dominated mainly by the meridional convergence of zonal momentum $\left(\xi_{3}\right)$ in the upper troposphere (Fig. 5c), in the middle troposphere, the vertical convergence term $\left(\xi_{4}\right)$ acts mainly to maintain the superrotating state (not shown). The acceleration associated with $\xi_{3}$ is confined in the upper troposphere and $\xi_{4}$ is responsible for spreading the westerly into the mid-troposphere. This result is also consistent with that of a previous study (KH05).

In $3 \mathrm{kw} 1$ experiment, which is the prototype for observations, the equatorial upper tropospheric $\overline{[u]}$ is westerly but is easterly in observation. This is likely due to lack of seasonality or hemispheric asymmetry in the model (Lee 1999; KH05). 

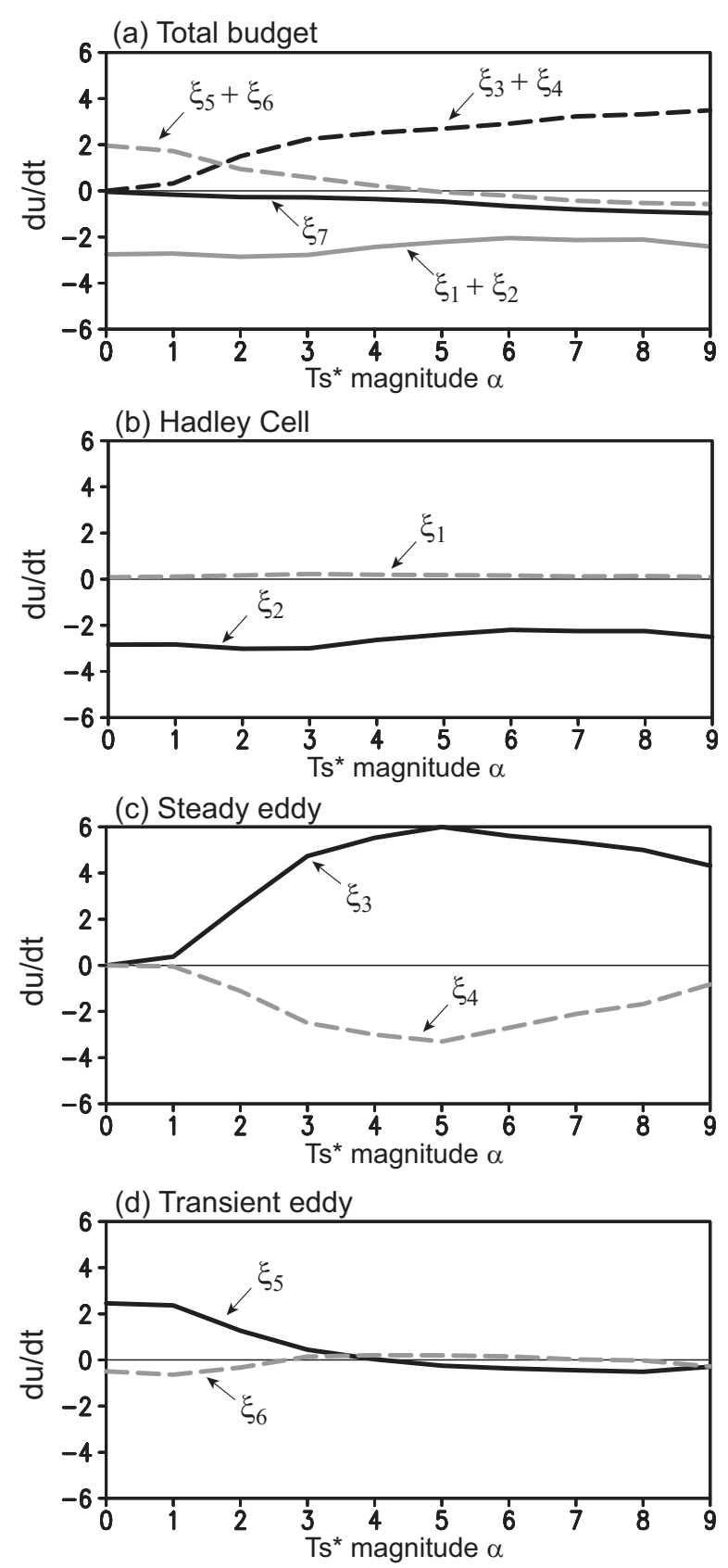

Fig. 5. The equatorial zonal momentum budget at 200hPa for each experiment: (a) all terms, (b) Hadley cell terms, (c) steady eddy terms, and (d) transient eddy terms. Zero on the horizontal axis denotes the CTL experiment. The unit of vertical axis is $1 \times 10^{-5} \mathrm{~m} \mathrm{~s}^{-2}$.

In the Northern Hemisphere, when Rossby waves forced at low-latitude propagate into a high-latitude region and are dissipated there, the waves tilting from northwest to southeast act to decelerate the zonal flow in the dissipation region, whereas they act to accelerate it in the source region. Hence, the acceleration of the equatorial zonal wind associated with the transient eddies in CTL indicates that the transient waves originate in the tropics, where they seem to be forced by transient heat sources.

In order to clarify what transient disturbance forces the Rossby waves at the equator, the Hovmöller diagrams of precipitation at the equatorial belt in CTL and $3 \mathrm{kw} 1$ are shown in Fig. 6. Note that the diagrams are duplicated in the longitudinal direction to clarify periodicity. No spatiotemporal filtering technique was used. It is evident that the eastward propagating structure is dominant in both figures, but the signal tends to be bounded over cold $T_{S}^{*}$ as $\alpha$ increases. The propagating speed in CTL and $3 \mathrm{kw} 1$ is approximately 13 $\mathrm{m} \mathrm{s}^{-1}$ and $12-15 \mathrm{~m} \mathrm{~s}^{-1}$ (the period is approximately 35 and 30-40 days), respectively. These characteristics indicate that this signal is a super cloud cluster (or supercluster, hereafter referred to as SCC; e.g., Hayashi and Sumi 1986; Nakazawa 1988).

We then perform a composite analysis to investigate whether the SCC actually forces the Rossby waves to propagate poleward. The composite procedure is as follows: First, for each day, we determine a longitude where the meridionally averaged $\left(5^{\circ} \mathrm{S}-5^{\circ} \mathrm{N}\right)$ precipitation anomaly (i.e., deviation from the longterm mean; denoted as $P^{\prime}$ ) has the maximum value. In many cases, the maximum corresponds to the SCC. Next, the map of $P^{\prime}$ and the corresponding variables for each day are moved in the longitudinal direction so that the maximum is located at the center of the map. Finally, the maps are composited (Fig. 7). In CTL, the composite for the streamfunction anomaly $\left(\psi^{\prime}\right)$ at the upper troposphere shows the wave train originating from the $P^{\prime}$ maximum region (Fig. 7a). Although part of the wave appears to be emitted from mid-latitude toward equator over the west of $P^{\prime}$ maximum, the largest equatorial convergence of zonal momentum flux associated with the transient waves $\left(u^{\prime} v^{\prime}\right)$ over slightly west of $P^{\prime}$ maximum (Fig. 7b) demonstrates that the waves tilt from northwest to southeast in the Northern Hemisphere, and the main source of the Rossby waves is diabatic heating associated with the SCC.

Nasuno (2008) performed aquaplanet experiments using a 7-km mesh AGCM, which includes explicit moist processes in a nonhydrostatic framework, with identical zonally uniform SST distribution. She showed that the upper tropospheric mean zonal wind is a weak westerly and is maintained by the momentum flux convergence associated with transient distur- 
(a) CTL

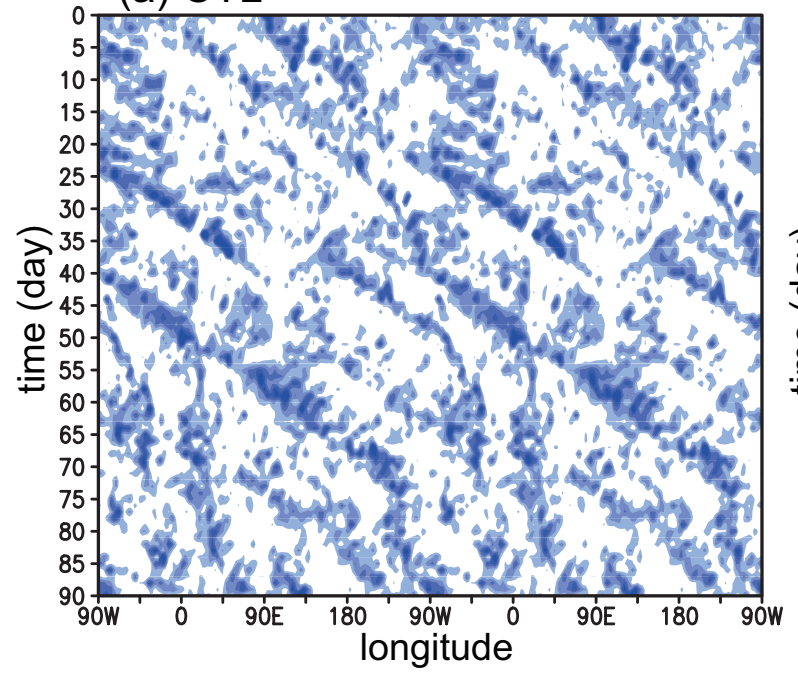

(b) 3kw1

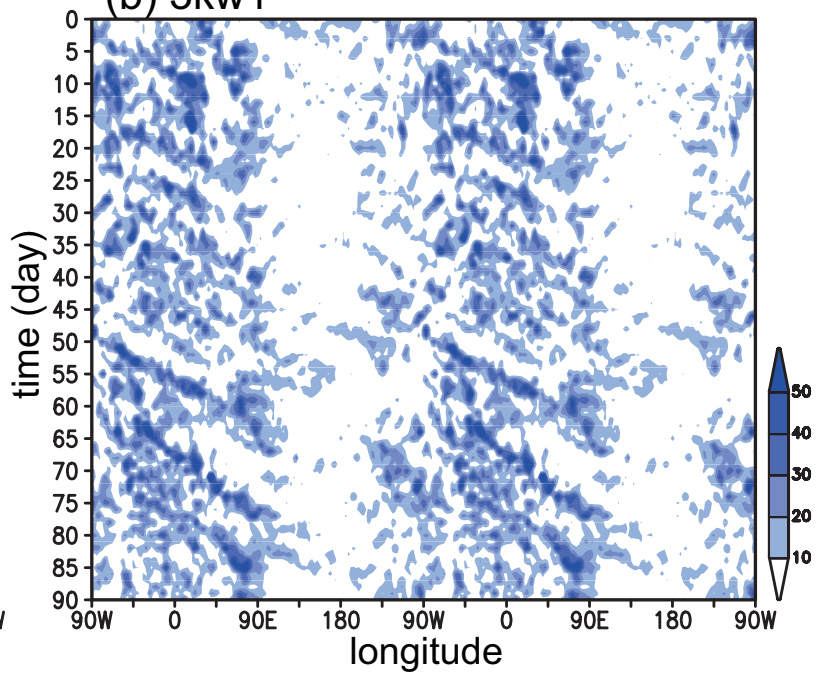

Fig. 6. Longitude-time sections of equatorial precipitation in (a) CTL and (b) $3 \mathrm{kw} 1$. The unit is $\mathrm{kg} \mathrm{m} \mathrm{m}^{-2} \mathrm{~d}^{-1}$. These figures are duplicated in the longitudinal direction.

bances; which accounted for moist Kelvin waves. This is consistent with our results, although no convective parameterization was used.

As $\alpha$ increases, the poleward propagating Rossby wave energy and the convergence of $u^{\prime} v^{\prime}$ at the equator are weakened (Figs. 7c-d), and the upper tropospheric westerly becomes to be maintained by the equatorward momentum transport associated with the steady waves (Fig. 5). When the zonally asymmetric SST is imposed, the poleward propagation of transient waves is prevented over warm $T_{S}{ }^{*}$ where the equatorial upper mean flow is easterly or weak westerly because of the off-equatorial anticyclonic steady response (not shown, but a similar indication is found in Fig. 3d). In addition, the reduction of the SCC over cold $T_{S}^{*}$ decreases the net convergence of $u^{\prime} v^{\prime}$ at the equator. Therefore, the momentum transport associated with the transients is reduced as $\alpha$ increases.

\section{Hadley cell}

\subsection{Response to tropical zonally asymmetric SST}

Hadley cell responses to the various SST distributions are described in this section. The right panels of Fig. 4 show the mean meridional streamfunction $\chi$ defined by Eq. (A8-9) in the CTL, 3kw1, and 9kw1 experiments. The comparison between CTL and 3kw1 shows that the strength of the Hadley cell is less in $3 \mathrm{kw} 1$ than in CTL by approximately $25 \%$. The Hadley cell is further weakened in $9 \mathrm{kw} 1$ without changes in the width and height of the cell.
Despite differences in the shape of the imposed SST or heating, the zonally asymmetric SST or heating that causes weakening of the Hadley cell similar to that seen in previous modeling studies (Suarez and Duffy 1992; Saravanan 1993; Inatsu et al. 2002; KH05). However, it is anticipated that the mechanisms may be different between the AGCMs including the precipitation processes and the dry dynamical models in which a heating distribution is imposed (Fig. 1). As stated in the introduction, the zonal-mean component of heating $[\bar{Q}]$ in AGCM is probably determined by the interaction with circulation via precipitation process even though the zonal-mean SST is kept constant throughout the experiments.

Indeed, $[\bar{Q}]$ significantly changes in the tropics when $\alpha$ is varied. Figure 8 shows the time- and zonalmean condensation $\left(\left[\overline{Q_{C}}\right]\right)$ and radiative heating $\left(\left[\overline{Q_{R}}\right]\right)$ distribution in the CTL and $3 \mathrm{kw} 1$ experiments. In the CTL experiment, $\left[\overline{Q_{C}}\right]$ is maximum at the equatorial mid-troposphere, while equatorial $\left[\overline{Q_{R}}\right]$ is weakly negative in the entire troposphere except for the upper troposphere (Figs. 8a, c). Both diabatic heating in $3 \mathrm{kw} 1$ obviously decrease in the equatorial middle to upper troposphere, and $\left[\overline{Q_{C}}\right]$ increases slightly in the off-equatorial (around $10^{\circ} \mathrm{S}$ and $10^{\circ} \mathrm{N}$ ) mid-troposphere (Figs. 8b, d).

The change in the Hadley cell influences the change in $[\bar{Q}]$ at the equator via precipitation processes such as water vapor transport, and vice versa. Therefore, it is conjectured that the weakening of the Hadley cell 

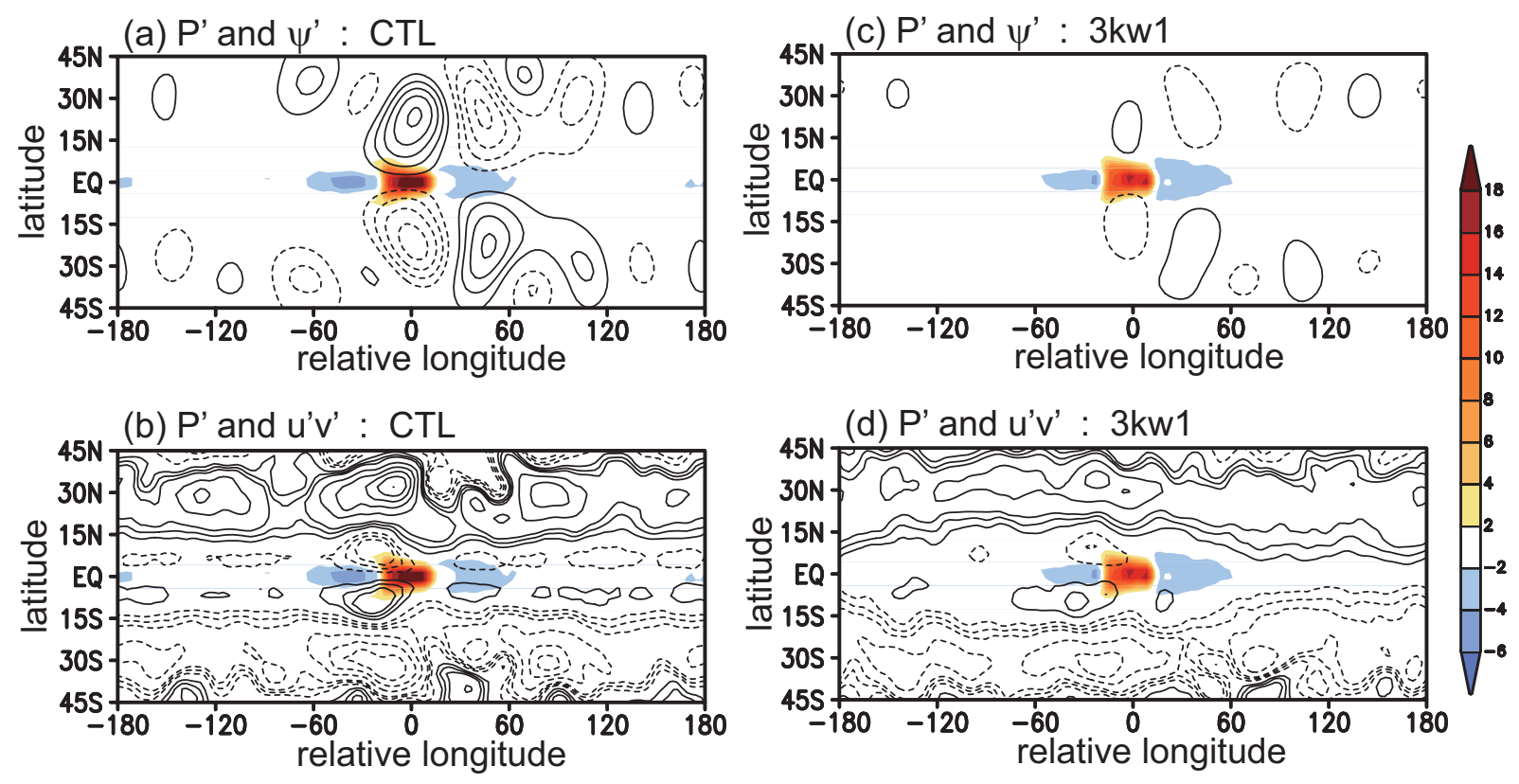

Fig. 7. Composite maps of transient disturbances in CTL: precipitation anomaly $P^{\prime}$ (shade: unit is kg m $\mathrm{m}^{-2} \mathrm{~d}^{-1}$ ) and (a) streamfunction anomaly $\psi^{\prime}$ at $200 \mathrm{hPa}$ (contour: interval is $3 \times 10^{6} \mathrm{~m}^{2} \mathrm{~s}^{-1}$ ) and (b) zonal momentum flux by transient eddy $u^{\prime} v^{\prime}$ at $200 \mathrm{hPa}$ (contour: interval is 10 within \pm 30 , otherwise, $30 \mathrm{~m}^{2} \mathrm{~s}^{-2}$ ). (c) and (d): same as (a) and (b) but in $3 \mathrm{kw} 1$. The zero contour is omitted and negative contours are dashed. The horizontal axis represents relative longitude from the $P^{\prime}$ maximum (denoted as 0 ).

primarily balances the reduction of $[\bar{Q}]$ at the equator. For this purpose, the contribution of each term to the Hadley cell is evaluated using a linear diagnostic equation for the mean meridional streamfunction:

$$
L \chi=\sum_{i=1}^{7} \eta_{i},
$$

where

$$
\begin{aligned}
& \eta_{1}={\overline{\left[Q_{C}\right.}}_{y}, \\
& \eta_{2}={\overline{\left[Q_{R}\right.}}_{y}, \\
& \eta_{3}=\left[\bar{u}^{*} \bar{v}^{*}\right]_{y p}+\left[\bar{u}^{*} \bar{\omega}^{*}\right]_{p p}, \\
& \eta_{4}=-\left[\bar{v}^{*} \bar{T}^{*}\right]_{y y}-\left[\bar{\omega}^{*} \bar{T}^{*}\right]_{y p}+\left[\bar{\omega}^{*} \bar{T}^{*}\right]_{y}, \\
& \eta_{5}={\overline{\left[u^{\prime} v^{\prime}\right]_{y p}}}+\overline{\left[u^{\prime} \omega^{\prime}\right]_{p p}}, \\
& \eta_{6}=-\overline{\left[\bar{v}^{\prime} T^{\prime}\right]_{y y}}-\overline{\left[\omega^{\prime} T^{\prime}\right]_{y p}}+\overline{\left[\omega^{\prime} T^{\prime}\right]_{y}}, \\
& \eta_{7}=-\overline{\left[F_{x}\right]_{p}}+\overline{\left[F_{T}\right]_{y}} .
\end{aligned}
$$

This equation is an alternate form of Eq. (A10). The elliptical linear operator is symbolically expressed as $L$, and $Q_{C}$ and $Q_{R}$ denote condensation and radiative heating (e.g., $Q_{C}=J_{C} / C_{p}$ ), respectively. The subscripts $y$ and $p$ denote derivatives with respect to latitude and pressure, respectively. This equation relates the mean meridional streamfunction $\chi$ to a seven source terms (right-hand side of Eq. (4)) and can be used to qualitatively diagnose the streamfunction response to the seven source terms. The first and second terms, $\eta_{1}$ and $\eta_{2}$, represent condensation and radiative heating, respectively, whereas $\eta_{3}$ and $\eta_{4}\left(\eta_{5}\right.$ and $\left.\eta_{6}\right)$ represent the forcing associated with steady (transient) eddy momentum and heat fluxes, respectively. The last term is frictional dissipation, which is calculated inside the AGCM.

All these source terms are calculated from the AGCM outputs of each experiment, and the $\chi$ response to each source term is obtained by solving Eq. (4). The responses are then averaged over $0^{\circ}-20^{\circ} \mathrm{N}$ and 1000 $150 \mathrm{hPa}$ and are plotted as deviations from the CTL experiment (Fig. 9). The streamfunction response to all source terms (black solid curve) shows that the Hadley cell is weakened as $\alpha$ increases. The decomposition of the $\chi$ response demonstrates that the weakening of responses to diabatic heating (gray solid and dashed curves) is obviously responsible for the weakening of the cell. This result is obtained because of the loose 
(a) condensation heating (CTL)

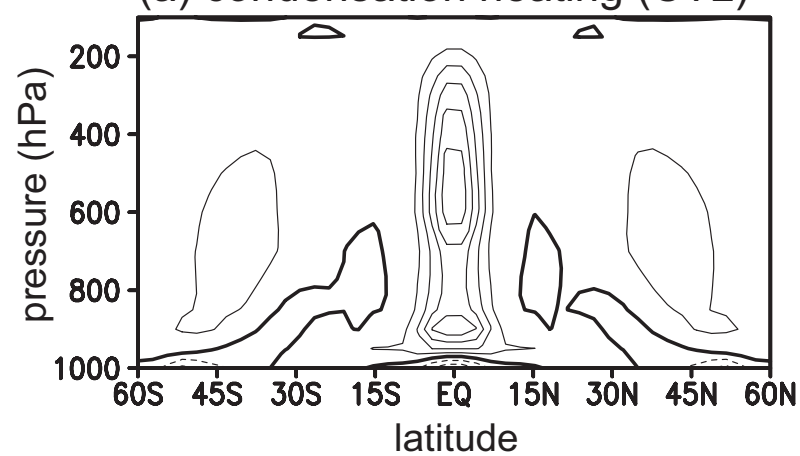

(b) condensation heating (3kw1)

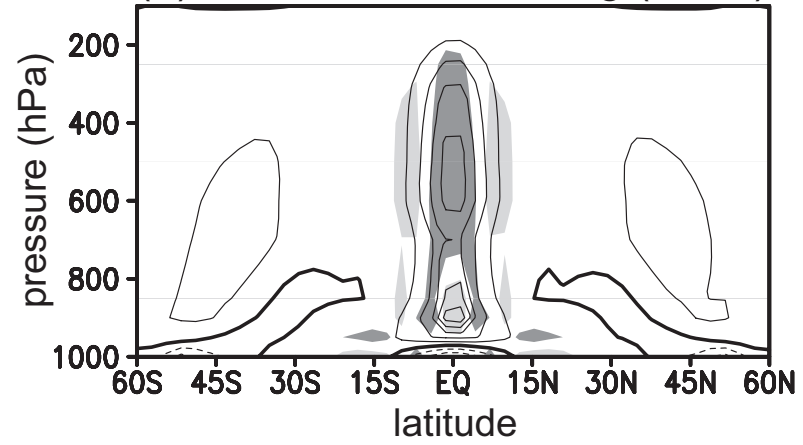

(c) radiative heating (CTL)

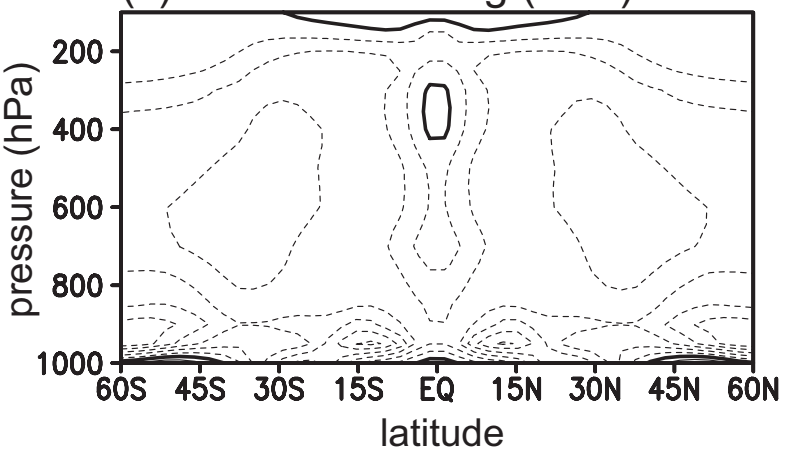

(d) radiative heating (3kw1)

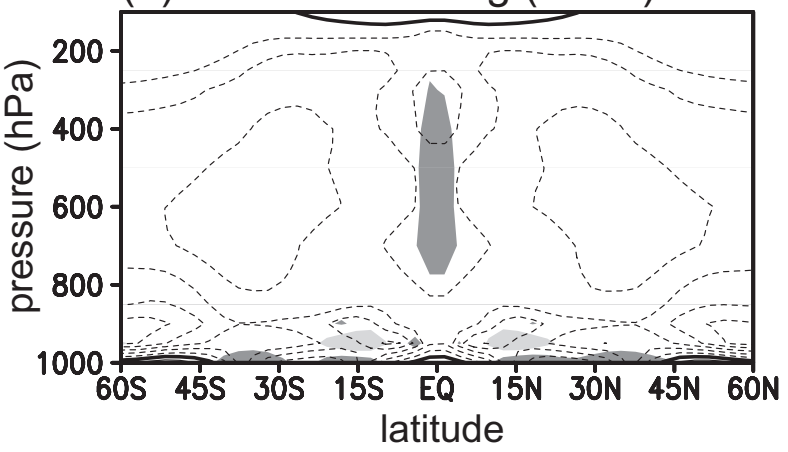

Fig. 8. Time and zonally averaged diabatic heating: condensation heating $\overline{\left[Q_{C}\right]}$ in (a) CTL and (b) $3 \mathrm{kw} 1$ and radiative heating $\overline{\left[Q_{R}\right]}$ in (c) CTL and (d) $3 \mathrm{kw} 1$. The contour interval is $1 \times 10^{-5} \mathrm{~K} \mathrm{~s}^{-1}$ in (a) and (b), and $0.5 \times 10^{-5}$ $\mathrm{K} \mathrm{s}^{-1}$ in (c) and (d). The zero contour is thickened and negative contours are dashed. The deviation from CTL in which the value is larger (smaller) than $0.3(-0.3) \times 10^{-5}$ is shaded as light (dark) gray in (b) and (d).

meridional gradient of heating (Fig. 8).

A notable feature is that the change in strength of the Hadley cell is not linearly proportional to $\alpha$ and the weakening is slightly counteracted when $\alpha$ is extremely large $(\alpha>7)$. This is mainly caused by the steady eddy terms, which show that both momentum and heat fluxes act to maintain the Hadley cell (black dotted curve). In particular, the first terms of $\eta_{3}$ and $\eta_{4}$ play a crucial role in the maintenance of $\chi$ in the upper and lower troposphere, respectively (not shown). However, the contribution from the steady eddies is small in $3 \mathrm{kw} 1$ which is the most realistic condition. The terms associated with the transient eddies are not important for the weakening of the cell (black dashed curve).

These results suggest that the weakening of the Hadley cell primarily balances the reduction of $[\bar{Q}]$ at the equator when $\alpha$ is not extremely large. It is plausible that the decreases of $\left[\overline{Q_{C}}\right]$ and $\left[\overline{Q_{R}}\right]$ at the equator reflect the decreases of precipitation and

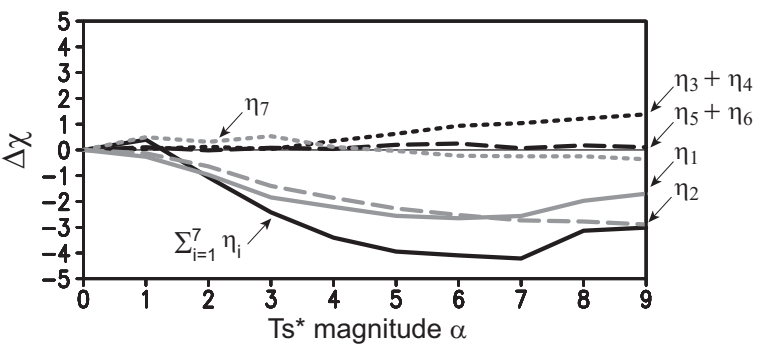

Fig. 9. The area averaged mean meridional streamfunction $\chi$ responses to some forcings diagnosed by Eq. (4). The deviation from CTL is plotted for each experiment. Zero on the horizontal axis denotes the CTL experiment. The unit of vertical axis is $1 \times 10^{3} \mathrm{~m} \mathrm{~s}^{-1} \mathrm{~Pa}$.

cloud, respectively. The change in convection will be analyzed in the next subsection. 


\subsection{Nonlinear relationship between precipitation and SST}

The time- and zonal-mean precipitation anomaly from CTL $(\Delta \overline{[P]})$ at the equator for each experiment is shown in Fig. 10 (black curve), where $\Delta$ denotes the deviation from the CTL. As anticipated from the above subsection, $\Delta \overline{[P]}$ decreases as $\alpha$ increases even though $\overline{\left[T_{S}\right]}$ is kept constant. However, the change is nonlinear because it increases for large $\alpha$.

To obtain insight into this change, $\Delta \overline{[P]}$ is divided into two contribution: one is the average over warm $T_{s}{ }^{*}$ and the other is that over cold $T_{s}^{*}$ (gray curves). Note that a size of area having warm $T_{S}^{*}$ is equivalent to that having cold $T_{s}^{*}$. The two curves illustrate that $\Delta \bar{P}$ over warm $T_{s}^{*}$ increases while that over cold anomaly decreases as $\alpha$ increases (see also Fig. 6). The zonalmean change $\Delta \overline{[P]}$ is the subtle difference between these two. Therefore, the decrease of equatorial $[Q]$ originates in the asymmetric precipitation response between over the warm and cold waters.

Next, we demonstrate that this asymmetry reflects a nonlinear relationship between precipitation and SST and present an evidence that the relationship is controlled by the large-scale atmospheric circulation. It is well known that the occurrence frequency of deep convection observed over the tropics dramatically increases when SST is above 27 or $28^{\circ} \mathrm{C}$ (e.g., Gadgil et al. 1984; Graham and Barnett 1987). A similar relationship is also detected in our AGCM experiments. Figures $11 \mathrm{a}, \mathrm{b}$ show the histogram of daily outgoing long-wave radiation (OLR) and precipitation over the tropics $\left(20^{\circ} \mathrm{S}-20^{\circ} \mathrm{N}\right)$ in the $3 \mathrm{kw} 1$ experiment. The histogram of OLR shows that the occurrence frequency of deep convection increases dramatically when SST is above $26-27^{\circ} \mathrm{C}$. Similarly, the rate of increase for the occurrence frequency of strong precipitation with respect to SST suddenly becomes large when SST is above $26-27^{\circ} \mathrm{C}$.

To discuss the change in the histogram from that of the CTL experiment, the equatorial OLR and precipitation are averaged for each bin of SST $\left(0.5^{\circ} \mathrm{C}\right.$ interval $)$ and are shown by black curves in Figs. 11a, b. Furthermore, the variables in CTL are also averaged, as depicted by black dots in Figs. 11a, b. It should be noted that the equatorial SST in CTL is zonally uniform at $27^{\circ} \mathrm{C}$, and the equatorial SST in $3 \mathrm{kw} 1$ ranges from 24 to $30^{\circ} \mathrm{C}$. Thus, the equatorial average for CTL $(3 \mathrm{kw} 1)$ is represented by a dot (a line). The comparison of this average for OLR between 3kw1 (curve) and CTL (dot) illustrates that the decrease of deep convection over cold $T_{S}^{*}\left(24^{\circ} \mathrm{C} \leq \mathrm{SST}<27^{\circ} \mathrm{C}\right)$ is larger than the increase over warm $T_{S}^{*}\left(27^{\circ} \mathrm{C} \leq \mathrm{SST} \leq 30^{\circ} \mathrm{C}\right)$. The

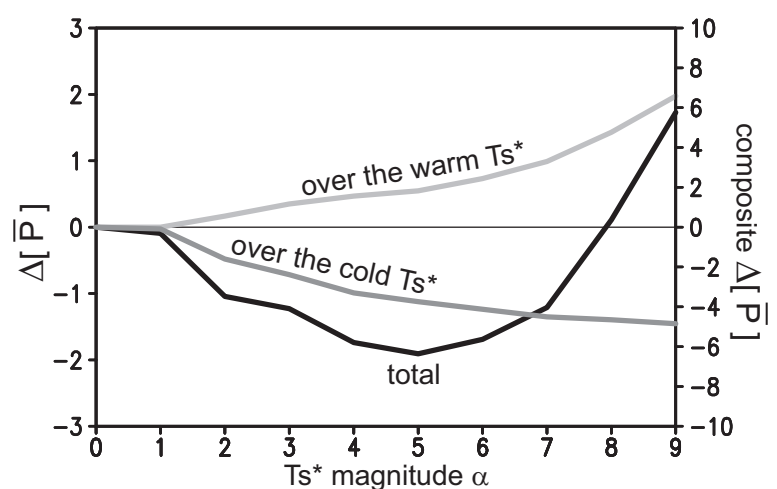

Fig. 10. The time and zonally averaged precipitation anomaly from CTL, $\Delta \overline{[P]}$, at the equator (black curve). Composited $\Delta \overline{[P]}$ over warm and cold $T_{s}^{*}$ are shown by gray curves, whose scale is shown to the right of the figure. Zero on the horizontal axis denotes the CTL experiment. The unit of vertical axis is $\mathrm{kg} \mathrm{m}^{-2} \mathrm{~d}^{-1}$.

precipitation also displays a similar relationship (Fig. $11 \mathrm{~b}$ ), that is, the decrease of precipitation over cold $T_{s}{ }^{*}$ is larger than the increase over warm $T_{s}^{*}$. Therefore, the zonally averaged precipitation (OLR), denoted as a black triangle, becomes smaller (larger) than that in CTL, which is denoted as a dot. If the rate of change for precipitation with respect to SST is constant, that is, it has a linear relationship, the increase in precipitation over warm $T_{s}{ }^{*}$ will be equal to the decrease over cold $T_{S}{ }^{*}$, and the zonal-mean precipitation will not change when a zonal wavenumber one $T_{S}^{*}$ is superimposed. Therefore, the decrease of $\overline{[P]}$ reflects the existence of the nonlinear relationship between precipitation and SST; it is dominated by the change in response of deep convection accompanying strong precipitation.

Then, what determines the nonlinear relationship? To gain insight into this question, the histograms of OLR and precipitation in the $6 \mathrm{kw} 1$ experiment are shown in Figs. 11c, d. In comparison with those of the $3 \mathrm{kw} 1$ experiment, the histograms and curves of OLR and precipitation in the $6 \mathrm{kw} 1$ obviously shift to a higher SST. If the nonlinearity is determined only by a local thermodynamic constraint between the SST and precipitation, the patterns of the histogram and curve are not changed. Therefore, this result indicates that an atmospheric effect plays a vital role in the nonlinear relationship. We will conclude in Section 4.4 that an interaction between large-scale circulation and deep convection such as a SCC determines the nonlinear relationship. 

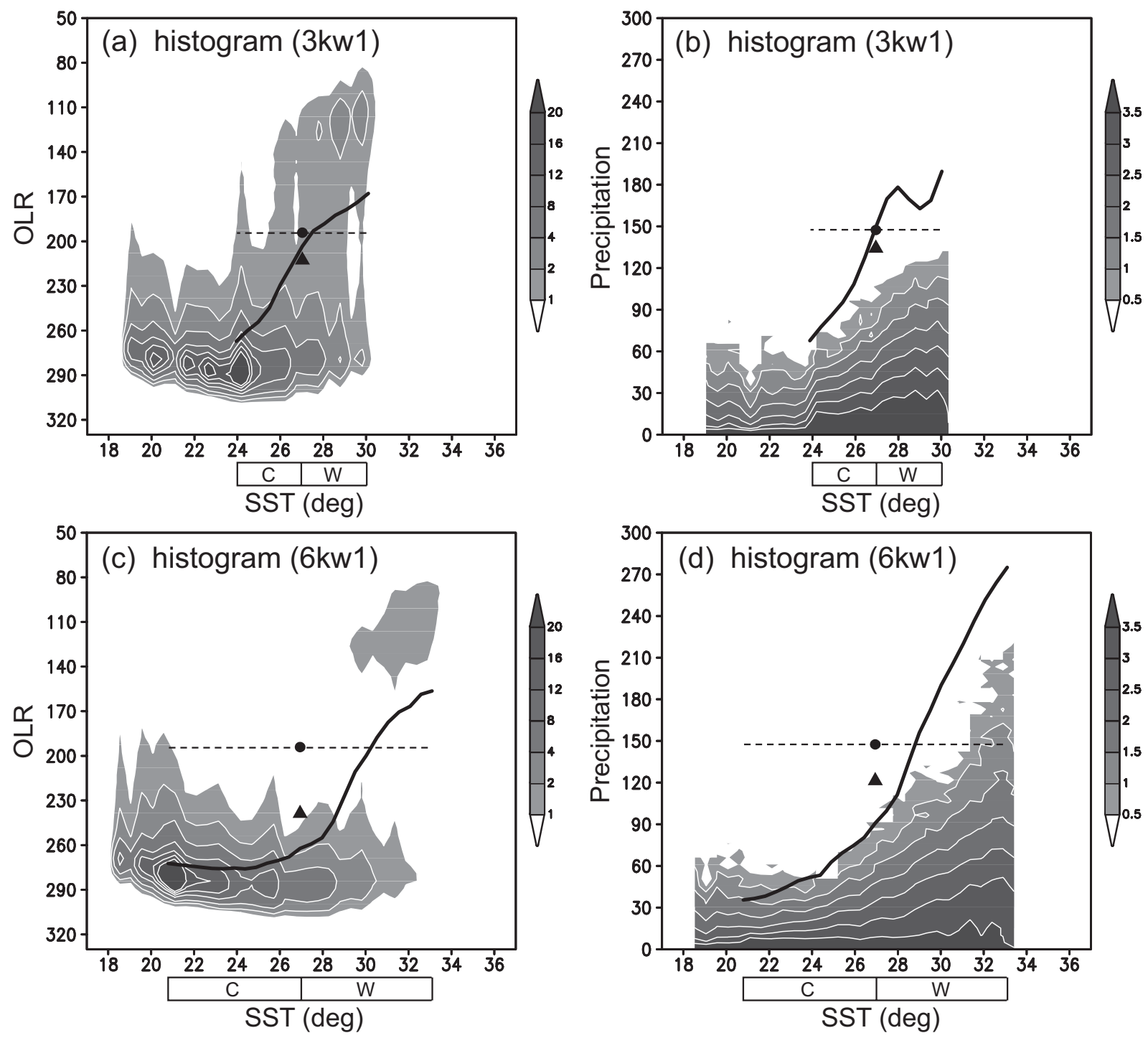

Fig. 11. Histograms of daily mean (a) OLR (scale is $1 \times 10^{3}$ ) and (b) precipitation (scale is power of 10) in $3 \mathrm{kw} 1$ over the tropics $\left(20^{\circ} \mathrm{S}-20^{\circ} \mathrm{N}\right)$. (c) and (d) Same as (a) and (b) but in $6 \mathrm{kw} 1$. The unit of the vertical axis of OLR is $\mathrm{W} \mathrm{m}{ }^{-2}$, and precipitation is $1 \times 10^{4} \mathrm{~kg} \mathrm{~m}^{-2} \mathrm{~s}^{-1}$. The interval of bin for SST is 0.5 , OLR is 5 , and precipitation is $5 \times 10^{4}$. The averaged equatorial OLR and precipitation for each bin of SST are represented by black curves. The time and zonally averaged OLR and precipitation in CTL and $3 \mathrm{kw1} / 6 \mathrm{kw} 1$ are depicted by black dot and triangle, respectively.

\subsection{Dynamic and thermodynamic changes in precip- itation}

To gain further insight into the nonlinear precipitation response with respect to $\mathrm{SST}, \Delta \bar{P}$ is separated into the "dynamic" and "thermodynamic" components following the procedure used in Bony et al. (2004) and Emori and Brown (2005). The time-averaged precipitation at a grid point can be represented by

$$
\bar{P}=\int_{-\infty}^{\infty} \operatorname{Pr}_{\omega} P_{\omega} d \omega
$$

where $P r_{\omega}$ is the probability density function of daily $500 \mathrm{hPa}$ vertical pressure velocity $\omega$ which is considered to be a proxy of the strength and/or frequency of a dynamic disturbance such as a SCC, and $P_{\omega}$ is the expected value of daily precipitation as a function of 
$\omega$. The latter is obtained by a composite of the daily precipitation for each $\omega$ bin. In this study, the bin width of $20 \mathrm{hPa} \mathrm{d}^{-1}$ is adopted. We have confirmed that following results are not sensitive to slight changes in the bin width.

The change in $\bar{P}$ between the $3 \mathrm{kw} 1$ and CTL experiments can be expressed as follows:

$$
\begin{aligned}
\Delta \bar{P}= & \int_{-\infty}^{\infty} \Delta \operatorname{Pr}_{\omega} P_{\omega} d \omega+\int_{-\infty}^{\infty} \operatorname{Pr}_{\omega} \Delta P_{\omega} d \omega \\
& +\int_{-\infty}^{\infty} \Delta \operatorname{Pr}_{\omega} \Delta P_{\omega} d \omega .
\end{aligned}
$$

The first term on the right-hand side is the change in $\bar{P}$ because of the change in the strength and/or frequency of the convective disturbance; therefore, this term is called "dynamic change." The second term occurs because of the change in the expected precipitation for a given $\omega$ and is regarded as a change caused by changes in an environment such as a moisture content. Hence, this term is called non-dynamic or "thermodynamic change." The third term is the covariance term.

The difference in deep tropical $\bar{P}$ between $3 \mathrm{kw} 1$ and CTL diagnosed by Eq. (6) is compared with that directly obtained from the model outputs (Fig. 12a). The diagnosed $\Delta \bar{P}$ is in good agreement with the correct value, indicating the adequacy of this analysis. The contributions from each term in the right-hand sides of Eq. (6) are shown in Fig. 12b. Both dynamic and thermodynamic changes illustrate that the decrease over cold $T_{S}^{*}$ is obviously larger than the increase over warm $T_{s}^{*}$. The covariance term is positive anywhere, but is distinguished only over cold $T_{S}^{*}$.

The change in $P r_{\omega}$ dominates the dynamic change. The occurrence frequency of ascent slightly increases over warm $T_{s}{ }^{*}$, whereas it significantly decreases over cold $T_{s}^{*}$ (Figs. 12c, d). These correspond to the slight increase in occurrence frequency of convection such as a SCC over warm $T_{s}^{*}$ and the extreme reduction over cold $T_{s}^{*}$ (see also Fig. 6b).

In contrast, the change in $P_{\omega}$ dominates the thermodynamic change. Because the occurrence frequency of extremely strong ascent in CTL is small (Figs. 12c, d), the change in precipitation is dominated by the change in $P_{\omega}$ with respect to a relatively small ascent (Figs. 12e, f). Such $\Delta P_{\omega}$ slightly increases over warm $T_{s}^{*}$, whereas it significantly decreases over cold $T_{s}{ }^{*}$. However, the thermodynamic change is almost zero over warm $T_{s}{ }^{*}$ and is confined over cold $T_{s}{ }^{*}$. Because of an environmental change, precipitation over cold $T_{s}^{*}$ is extremely less even if the occurrence frequency of convection is the same as that in CTL.

These results indicate that both dynamic and thermo- dynamic changes contribute to the asymmetric precipitation responses between over warm and cold $T_{s}^{*}$, that is, the nonlinear relationship between precipitation and SST. As shown in the above subsection, not only the imposed SST anomaly but also an atmospheric circulation change seems to affect the extreme reduction of convection frequency and the amount of precipitation over cold $T_{s}{ }^{*}$. The change in atmospheric mean circulation from CTL is investigated in the next subsection.

\subsection{Change in atmospheric mean circulation}

Figures $13 \mathrm{a}-\mathrm{c}$ show the deep tropical cross section of zonal-mean plus eddy relative humidity $(\Delta \overline{R h})$ and $\Delta \bar{\omega}$, the sum of $\Delta \overline{Q_{c}}$ and $\Delta \overline{Q_{R}}(\Delta \bar{Q})$ and static stability $\left(\Delta \overline{S_{p}}\right)$, and $\Delta \overline{Q_{c}}$ and $\Delta \overline{Q_{R}}$, respectively, for $3 \mathrm{kw} 1$. As anticipated from the negative (positive) $\Delta \overline{[P]}(\Delta \overline{[O L R]})$ shown in Section 4.2, the responses for both $\Delta \overline{Q_{c}}$ and $\Delta \overline{Q_{R}}$ are zonally asymmetric (Fig. $13 \mathrm{c})$. In the middle to upper troposphere, the magnitude of the cooling anomaly over cold $T_{s}^{*}$ is larger than that of the heating anomaly over warm $T_{s}^{*}$, and the zonally averaged $\Delta \bar{Q}$ is negative in the deep tropics (see also Figs. 8b, d). This reflects the distinct decrease of deep convection over cold $T_{s}^{*}$. The pattern of $\Delta \bar{Q}$ is very similar to that of $\Delta \bar{\omega}$, which indicates that the change in $\bar{Q}$ is almost balanced by the change in $\bar{\omega}$ in the deep tropics (shading in Figs. 13a, b). The heat budget analysis also supports this result (not shown), in which the following balance relation approximately applies:

$$
\Delta \bar{Q} \simeq-\overline{S_{p}} \Delta \bar{\omega} .
$$

We confirmed that the term $-\bar{\omega} \Delta \overline{S_{p}}$ and transient eddy terms are sufficiently smaller than others, and $\Delta \bar{Q}$ is mainly composed of $\Delta \overline{Q_{c}}$ (Fig. 13c), where $\bar{\omega}$ and $\overline{S_{P}}$ in CTL are zonally uniform (not shown). Thus, the response of $\Delta \bar{\omega}$ is also zonally asymmetric and the magnitude of the descending anomaly over cold $T_{S}^{*}$ is larger than that of the ascending anomaly over warm $T_{s}^{*}$ in the middle to upper troposphere (Fig. 13a). This asymmetric magnitude implies the weakening of the Hadley cell as seen in $\Delta \overline{[\omega]}^{2}$. Therefore, these results indicate that the weakening of the Hadley cell is equivalent to the decrease of $[\bar{Q}]$. This is consistent with the result of the linear diagnostic analysis in Section 4.1.

The weakening mechanism of the Hadley cell is then interpreted as follows: When the $3 \mathrm{kw} 1 \mathrm{SST}$ anomaly is

\footnotetext{
${ }^{2}$ It should be noted that the longitudinal width of the descending anomaly is slightly larger than that of the ascending anomaly. This point will be discussed in the last paragraph of this subsection.
} 

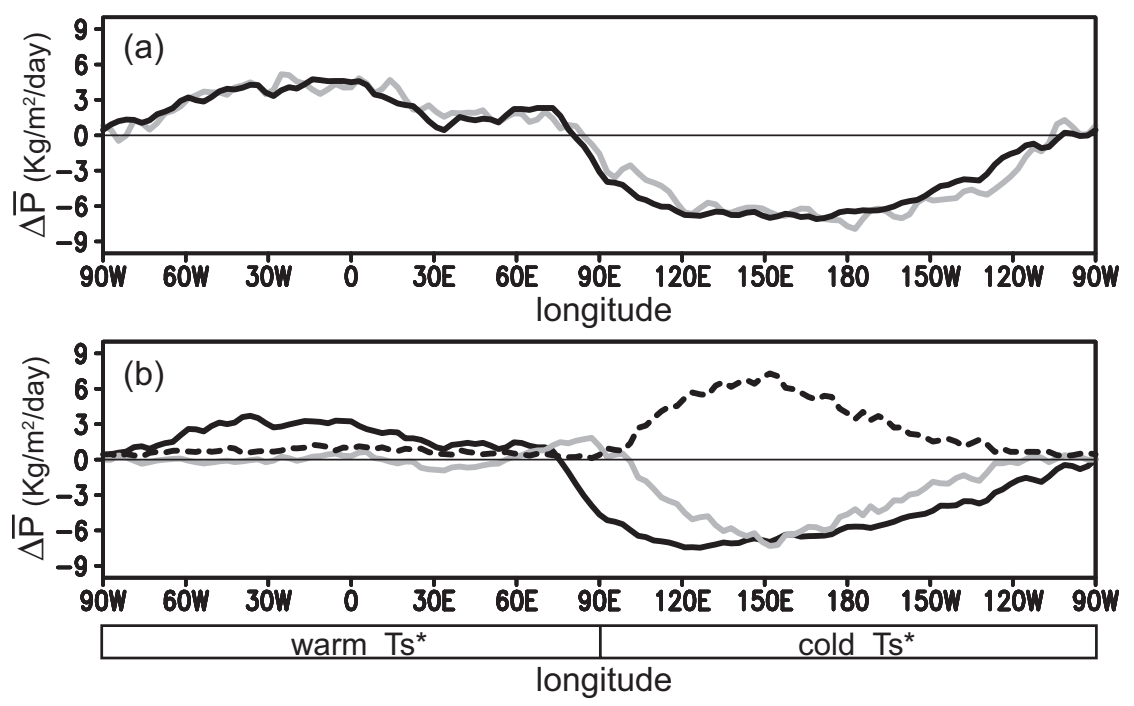

(c) Prw over the warm Ts*

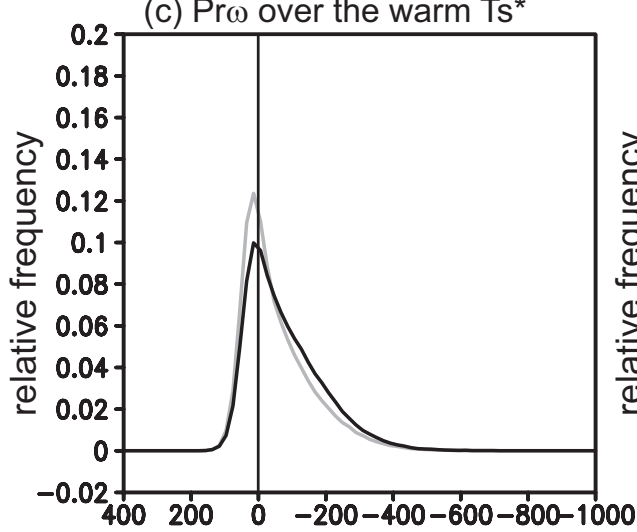

(d) Prw over the cold Ts*

$\omega$ (hPa/day)

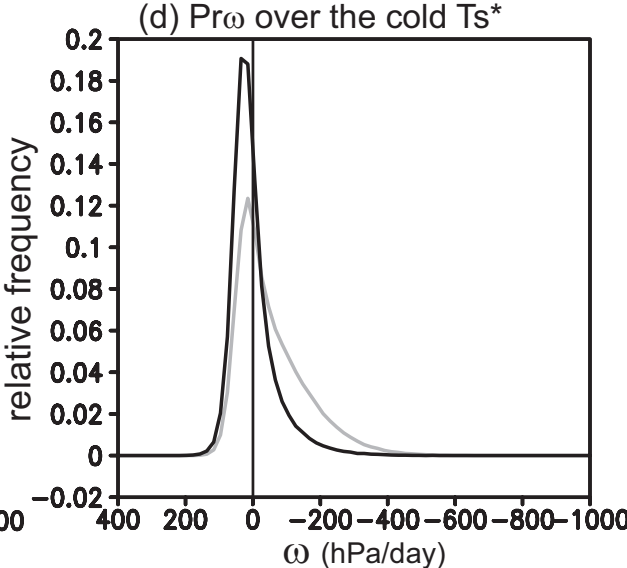

(e) P $\omega$ over the warm Ts*
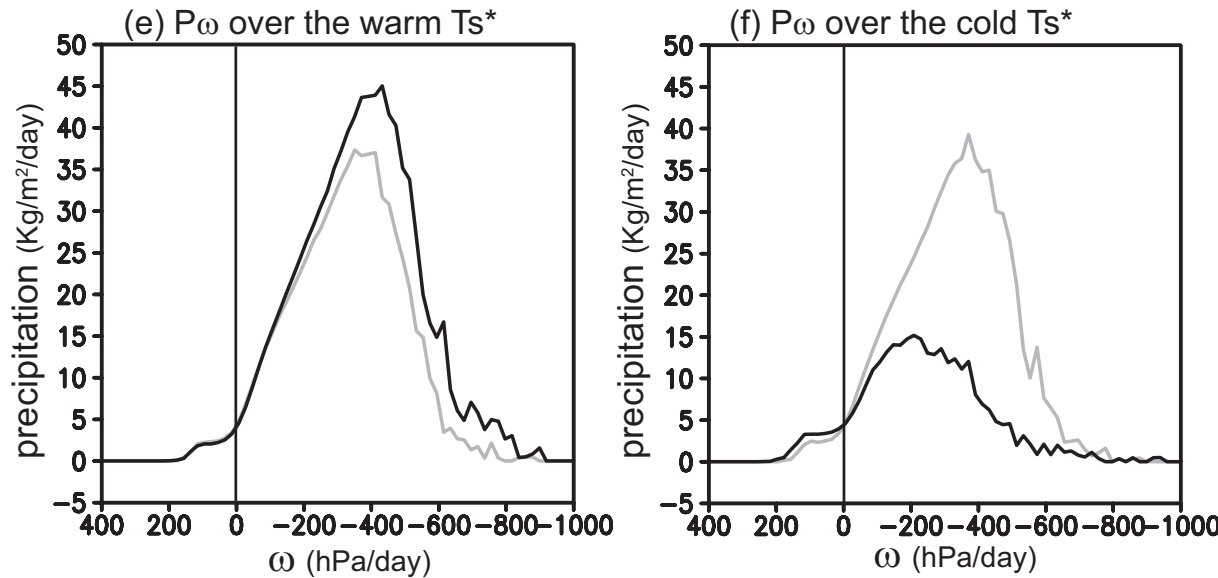

Fig. 12. The change in tropical (averaged over $5^{\circ} \mathrm{S}-5^{\circ} \mathrm{N}$ ) mean precipitation between $3 \mathrm{kw} 1$ and $\mathrm{CTL}, \Delta \bar{P}$; (a) diagnosed by Eq. (6) (black solid line) and obtained by model output (gray solid line), (b) due to the dynamic (black solid line), the thermodynamic change (gray solid line), and the cross term (black dotted line). (c) $\operatorname{Pr}_{\omega}$ in CTL (gray line) and $3 \mathrm{kw} 1$ (black line) averaged over warm $T_{S}{ }^{*}$ over the tropics $5^{\circ} \mathrm{S}-5^{\circ} \mathrm{N}$, (d) same as (c) but averaged over cold $T_{s}^{*}$, (e) same as (c) but $P_{\omega}$, (f) same as (d) but $P_{\omega}$. 
(a) $\Delta \bar{\omega} \& \Delta \overline{\mathrm{Rh}}$ in $3 \mathrm{kw} 1$

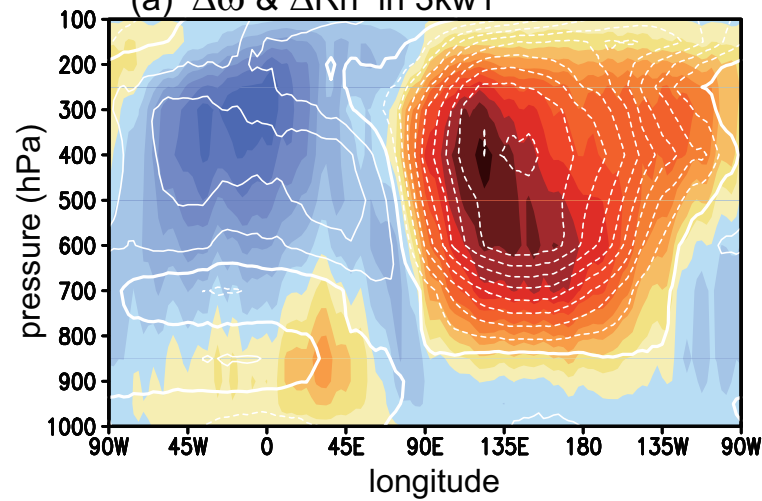

(b) $\Delta \bar{Q} \& \Delta \overline{S p}$ in $3 k w 1$

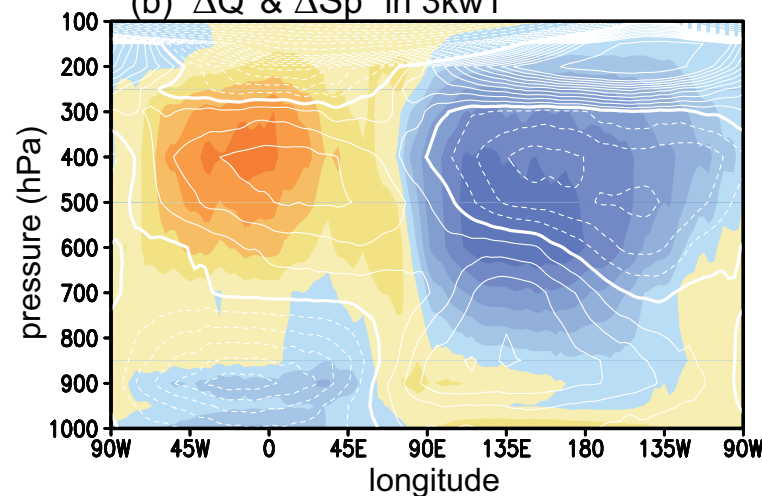

(c) $\Delta \bar{Q}_{C} \& \Delta \bar{Q}_{R}$ in $3 k w 1$

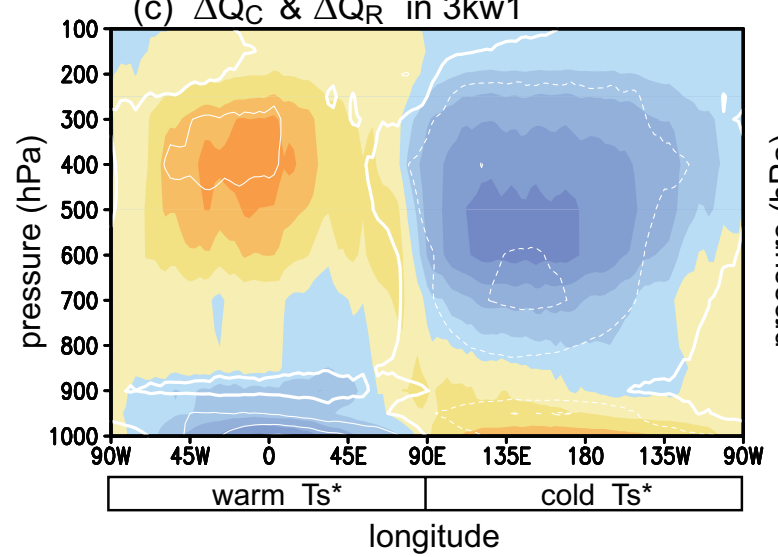

(d) $\Delta \bar{\omega} \& \Delta \overline{\mathrm{Rh}}$ in $6 \mathrm{kw} 1$

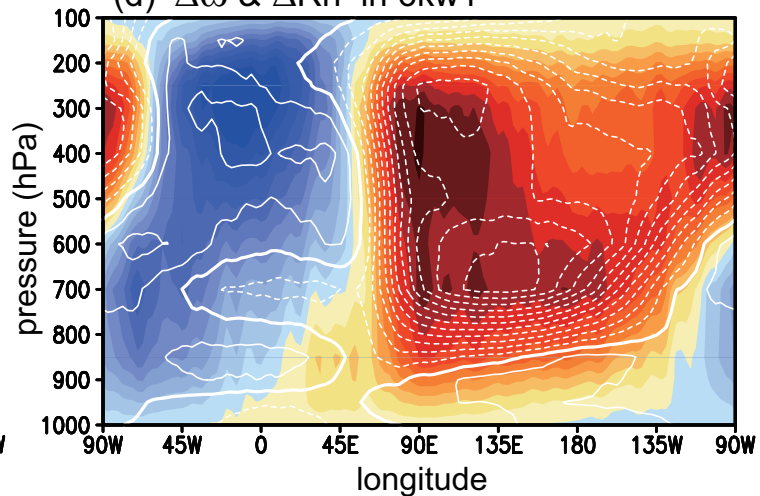

(e) $\Delta \bar{Q} \& \Delta \overline{S p}$ in $6 k w 1$

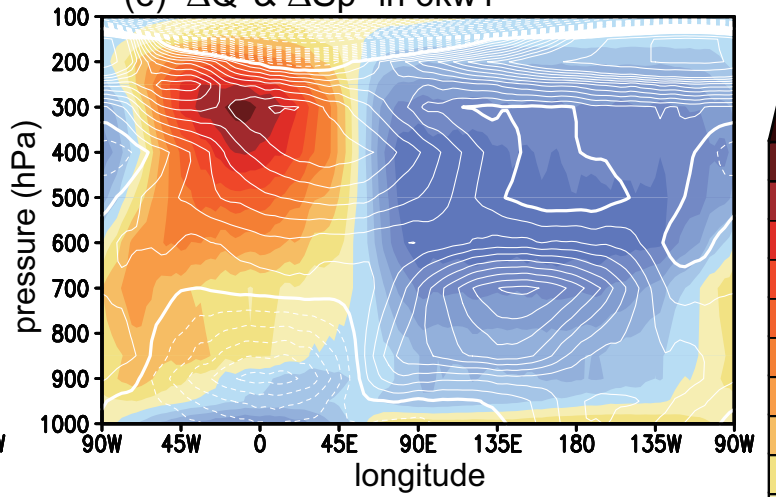

(f) $\Delta \bar{Q}_{C} \& \Delta \bar{Q}_{R}$ in $6 k w 1$

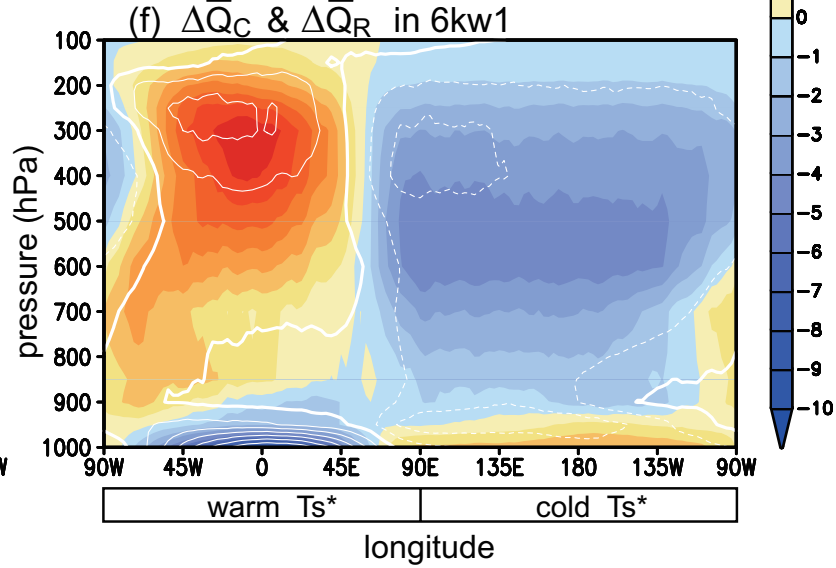

Fig. 13. Temporary and meridionally $\left(5^{\circ} \mathrm{S}-5^{\circ} \mathrm{N}\right)$ averaged vertical structure of the anomaly (deviation from CTL) in $3 \mathrm{kw1}$ : (a) vertical pressure velocity (shade: unit is $1 \times 10^{-4} \mathrm{hPa} \mathrm{s}^{-1}$ ) and relative humidity (contour: interval is 0.05 ), (b) diabatic heating (shade: $1 \times 10^{-5} \mathrm{~K} \mathrm{~s}^{-1}$ ) and static stability (contour: interval is $0.3 \times 10^{-4}$ within $\pm 3 \times$ $10^{-4}$, otherwise, $1 \times 10^{-4} \mathrm{~K} \mathrm{~Pa}^{-1}$ ). (c) condensation heating (shade) and radiative heating (contour), scales are the same with the diabatic heating in (b). (d)-(f) are the same as (a)-(c) but in 6kw1. The zero contour is thickened and negative contours are dashed.

superimposed on CTL SST, the anomaly accompanies the zonal SST gradient over the tropics, which induces the lower tropospheric winds blowing from the cold
$T_{S}^{*}$ region. The wind convergence (divergence) over warm (cold) $T_{s}^{*}$ should promote an upward (downward) flow anomaly there; thus, the Walker circula- 
tion with one cell is excited. The similarity of pattern between $\Delta \bar{\omega}$ and $\Delta \overline{R h}$ (Fig. 13a) implies that the ascending (descending) anomaly acts to enhance (suppress) the upward water vapor transport, and $R h$ increases (decreases) in the middle to upper troposphere. At the same time, the upward (downward) flow anomaly and the warm (cold) boundary condition induce the decrease (increase) of $S_{p}$ in the lower troposphere (Fig. 13b). These changes in $R h$ and $S_{p}$ act to enhance (suppress) the occurrence of deep convection and the amount of precipitation over warm (cold) $T_{s}{ }^{*}$.

However, the response of precipitation is zonally asymmetric. The change in $\bar{\omega}, \mathrm{Rh}$ and $S_{p}$ would more actively affect the change in precipitation over cold $T_{S}{ }^{*}$ (dynamic and thermodynamic change). Because an atmospheric column over warm $T_{s}^{*}$ is sufficiently wet and unstable for deep convection even in CTL, the increase of precipitation over warm $T_{s}^{*}$ is not very large. In contrast, over cold $T_{s}{ }^{*}$, the increase of dry mass in the middle to upper troposphere and the increase of stability in the lower troposphere act to dramatically suppress the occurrence of deep convection and the amount of precipitation. Hence, the decrease of $Q$ over the cold $T_{S}{ }^{*}$ is larger than the increase over warm $T_{S}{ }^{*}$ in the middle to upper troposphere.

At the same time, to satisfy the balance equation (7), the increase of $\bar{\omega}$ over cold $T_{s}^{*}$ must be larger than the decrease over warm $T_{s}^{*}$. This change in large-scale circulation feeds back to a further change in $R h$ and $S_{p}$. Consequently, the asymmetric response of $P, Q_{C}$, and $Q_{R}$ between over warm and cold $T_{S}^{*}$ is further reinforced. Therefore, this interaction between the atmospheric large-scale circulation and deep convection determines the nonlinear relationship between precipitation and SST and controls the zonal asymmetry of $\Delta \bar{Q}$ and $\Delta \bar{\omega}$, that is, the weakening of the Hadley cell. Figures $13 \mathrm{a}-\mathrm{c}$ illustrate a final balance state in which this positive feedback is settled.

It is useful to mention the contribution of the zonal expansion of subsidence anomaly to the weakening of the Hadley cell. Figure 12a shows that the negative $\Delta \bar{P}$ spreads slightly into the eastern side of the warm SST anomaly. This is caused by dynamic change (Fig. 12b), which relates to the slight expansion of the subsidence anomaly into the warm $T_{s}^{*}$ region (Fig. 13a). In $3 \mathrm{kw} 1$, the contribution of this expansion to the slight increase of $\Delta \bar{P}$ over warm $T_{s}^{*}$ is small. In contrast, in $6 \mathrm{kw} 1$, we may not be able to ignore this effect on the weakening of the cell. Figures $13 \mathrm{~d}-\mathrm{f}$ demonstrate that positive $\Delta \bar{\omega}$ accompanying negative $\Delta \bar{Q}$ in the troposphere spreads over the eastern side of warm $T_{S}^{*}$. This indicates that $\Delta \bar{P}$ is negative in this region even though the SST is warmer than $27^{\circ} \mathrm{C}$ and this change in circulation is responsible for the histogram shift shown in Fig. 11. Therefore, when $\alpha$ is relatively large, we may need to consider the expansion of the longitudinal width of the subsidence anomaly for the weakening of the Hadley cell. However, determining what controls the width is very difficult (e.g., Pierrehumbert 1995) and is beyond the scope of this study.

\subsection{Moist static energy budget}

To discuss the experimental results from another view point, the moist static energy (MSE) balance is examined (e.g., Numaguti 1993; Satoh 1994; Frierson 2007). The vertically integrated budget of time- and zonal-mean MSE is expressed as

$$
\frac{1}{a \cos \varphi} \frac{\partial}{\partial \varphi}\langle\overline{\langle h \nu]}\rangle \cos \varphi=\langle\overline{[R]}\rangle+\overline{[L E]}+\overline{[H]},
$$

where $\langle A\rangle \equiv \int_{0}^{z_{t}} \rho A d z$ represents the vertical integration from the surface to the top of the atmosphere (TOA), $z_{t}$, and $h$ is MSE, defined as the sum of enthalpy, potential energy, and latent energy; $h=C_{p} T$ $+g z+L q, \rho$ the density of the air, $L$ the latent heat of condensation, $E$ the evaporation from the surface, $H$ the sensible heat flux form the surface, $\langle R\rangle=R^{T}-R^{S}$ is the heating rate by radiative process, $R^{T}$ and $R^{S}$ are the net downward radiation at the TOA and the surface respectively, and $R^{T}$ is decomposed short- and longwave radiation; $R^{T}=R_{S}^{T}+R_{L}^{T}$. The MSE flux is decomposed into stationary mean flow term, stationary eddy term, and transient eddy term; $\langle\overline{[h v]}\rangle=\langle[\bar{h}][\bar{v}]\rangle+$ $\left\langle\left[\bar{h}^{*} \bar{v}^{*}\right]\right\rangle+\left\langle\left[\overline{h^{\prime} v^{\prime}}\right]\right\rangle$.

Figures $14 \mathrm{a}, \mathrm{b}$ show the distribution of net MSE flux and net energy supply (right-hand side of Eq. 8) in the CTL experiment. The imbalance of energy supply between the tropics and the high-latitudes is compensated with poleward energy transport through the atmosphere. Because of the absence of a stationary eddy, the poleward MSE transport is accomplished only by mean flow and transient eddies. This energy balance is changed slightly when the zonal wavenumber one SST anomaly is superimposed. The difference in these fluxes between $3 \mathrm{kw} 1$ and CTL are shown in Figs. 14c, d. In 3kw1, the net energy supply decreases over the tropics and increases over the subtropics ${ }^{3}$. Corresponding to this change, the net poleward MSE transport decreases over the Hadley cell region and increases over the mid-latitudes. The decomposition of

\footnotetext{
${ }^{3}$ The time- and global-mean net energy supply is almost zero in both experiments: both experiments are in a state of energy equilibrium.
} 
(a) MSE flux in CTL

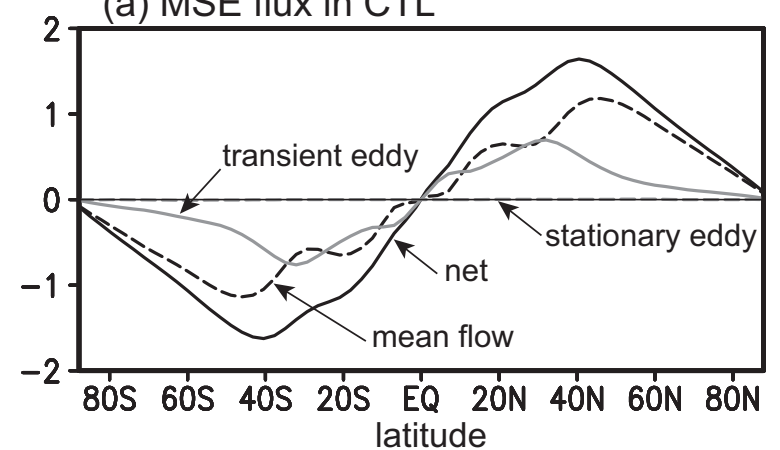

(c) MSE flux diff. (3kw1 - CTL)
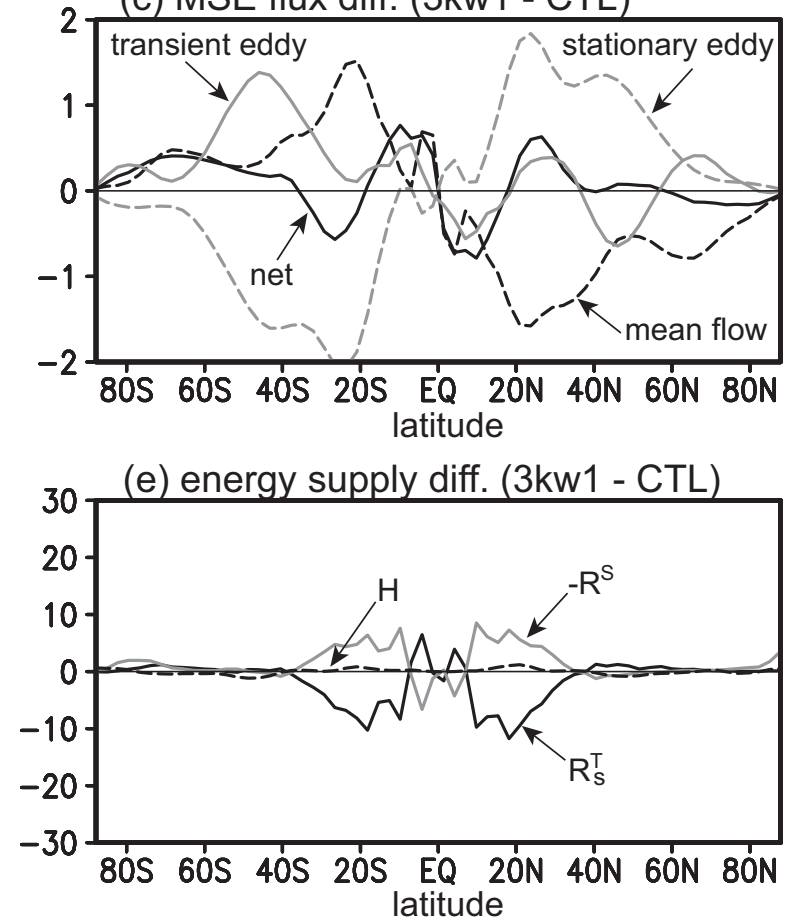

(b) net energy supply in CTL

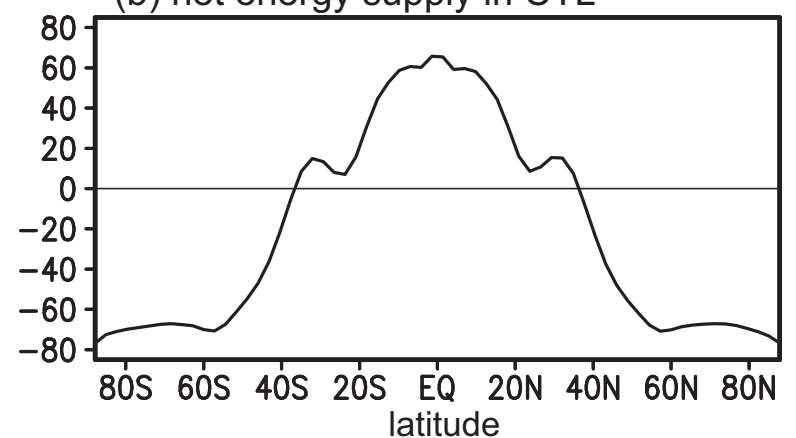

(d) net energy supply diff. (3kw1 - CTL)
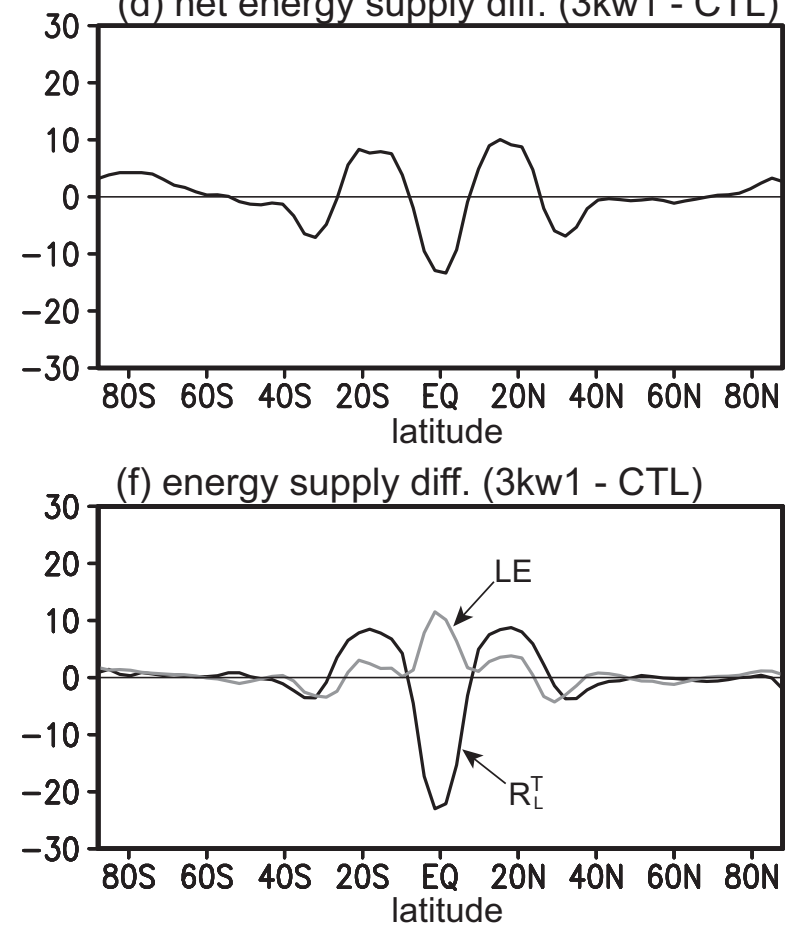

Fig. 14. (a) The meridional MSE flux (unit is $1 \times 10^{8} \mathrm{~W} \mathrm{~m}^{-1}$ ) and (b) the net energy supply (unit is $\mathrm{W} \mathrm{m} \mathrm{m}^{-2}$ ). (c) The MSE flux difference between $3 \mathrm{kw} 1$ and CTL (unit is $1 \times 10^{7} \mathrm{~W} \mathrm{~m}^{-1}$ ). The energy flux difference between $3 \mathrm{kw} 1$ and CTL: (d) the net energy supply, (e) $\overline{[H]}, \overline{\left[R_{S}^{T}\right]}$ and $-\overline{\left[R^{S}\right]}$, (f) $\overline{[L E]}$ and $\overline{\left[R_{L}^{T}\right]}$.

the MSE flux difference demonstrates that the mean flow term and the transient eddy term are dominant in the decrease over the Hadley cell region, whereas the stationary eddy term acts to increase poleward transport. As mentioned in the last paragraph of Section 3 , when the zonally asymmetric SST is imposed, the stationary eddy over warm $T_{S}^{*}$ acts to prevent the poleward propagation of transient waves and the transient heat source associated with deep convection tends to decrease over cold $T_{s}^{*}$. As a result, the poleward MSE flux caused by transient eddies decreases, whereas that caused by stationary eddies increases over the Hadley cell region. The decrease of poleward flux by mean flow corresponds to the weakening of the Hadley cell.

Now let us examine the change in terms of energy supply (Figs. 14d-f). The change in incoming solar radiation $\left(R_{S}^{T}\right)$ and surface radiation flux $\left(R^{S}\right)$ tend to cancel each other (Fig. 14e). The dominant change in the tropics is the increase of evaporation and decrease of $R_{L}^{T}$ (that is, an increase of OLR): the decrease of net energy supply originates in the small difference between these two (Fig. 14f). These results indicate 
that the weakening of the Hadley cell corresponds mainly to the increase of OLR over the tropics, which is consistent with the results discussed in the previous section.

The weakening mechanism of the Hadley cell is explained from energetics as follows: When the zonal wavenumber one SST anomaly is added, the Walker circulation is excited. The occurrence frequency of deep convection over cold $T_{s}{ }^{*}$ is effectively suppressed by the dry subsidence anomaly and the positive static stability anomaly in the lower troposphere. The OLR increases over cold $T_{s}^{*}$ because of the increase in clear sky (Fig. 6b). The enhancement of deep convection over warm $T_{s}^{*}$ is not very large because the atmosphere is sufficiently wet and unstable even in CTL. Hence, the change in time- and zonal-mean OLR is positive, and the energy that escapes into outer space increases over the tropics. Furthermore, the stationary eddies forced by the SST anomaly transports energy from the tropics poleward. The lack of energy over the tropics cannot be compensated by only the decrease of poleward energy transport by the transient eddies because its activity is originally low in this region. Therefore, the poleward transport by mean flow must also decrease to satisfy the energy balance, and the strength of the Hadley cell is weakened.

Often we think that the radiative cooling in the subtropics drives the Hadley cell, but in this case, the cell strength is controlled by the radiative cooling in the tropical belt of the upward motion region of the Hadley circulation.

\section{Model intercomparison}

In this section, we compare the results of our AGCM (referred to as "K1-JAPAN" in this section) with that of other 15 AGCMs integrated with identical SST distribution to discuss the robustness of our results and the causes of inter-model differences (see Williamson et al. 2012, 2013 for detailed description of these models). The CTL and $3 \mathrm{kw} 1$ experiments of $16 \mathrm{AGCM}$ outputs submitted to APE are used. Hereinafter, all variables are temporally, zonally, and meridionally $\left(5^{\circ} \mathrm{S}-5^{\circ} \mathrm{N}\right)$ averaged unless stated otherwise.

First, we focus on superrotation. As shown in Section 3, the equatorial time- and zonal-mean zonal wind in the upper troposphere is a weak westerly in our CTL experiment, and the westerly is strengthened as $\alpha$ increases. To examine inter-model differences, a scatter diagram is shown in Fig. 15a. The x-axis is averaged $200 \mathrm{hPa}$ zonal wind in CTL $\left(\overline{[u}_{\mathrm{ctl}}\right)$ and the $\mathrm{y}$-axis represents the difference between $3 \mathrm{kw} 1$ and CTL $(\Delta[u])$. It is found that $\overline{[u}_{\mathrm{ctl}}$ is westerly in some AGCMs but easterly in the others. Nevertheless, the superrotation occurs in all AGCMs when the zonally asymmetric SST anomaly is given, although the strength is different among AGCMs. These indicate that our results are not unique, and the difference in physical parameterization yields a significant inter-model difference in the polarity of $\overline{[u}_{\mathrm{ctl}}$ and the strength of $\Delta \overline{[u]}$.

Figures $15 \mathrm{~b}$, c show the scatter diagram for mean meridional streamfunction difference $(\Delta \chi)^{4}$ against the mean precipitation difference $(\Delta \overline{[P]})$ and mean OLR difference $(\Delta \overline{[\mathrm{OLR}]})$, respectively. The first figure demonstrates that the Hadley cell weakens and the mean tropical precipitation decreases in all AGCMs when the zonally asymmetric SST anomaly is added, though the strength is different among AGCMs. The weakening of the Hadley cell is roughly proportional to $\Delta \overline{[P]}$, and the correlation coefficient between $\Delta \chi$ and $\Delta \overline{[P]}$ is 0.55 , which is significant at the $95 \%$ confidence level. When we eliminate LASG, which is a model that does not conserve the global atmospheric water budget (not shown), the correlation coefficient becomes 0.75 and is significant at the $99 \%$ confidence level.

The decrease of $\Delta \overline{[P]}$ seems to mainly correspond to the effective suppression of deep convection also in other AGCMs. Because $\Delta \overline{[\mathrm{OLR}]}$ increases in all AGCMs (Fig. 15c) and the correlation coefficient between $\Delta \overline{[P]}$ and $\Delta \overline{[\mathrm{OLR}]}$ reaches $-0.66(-0.30)$ which is significant at the $99 \%$ confidence level (not significant) without (with) LASG. Figure $15 \mathrm{c}$ shows that $\Delta \overline{[\mathrm{OLR}]}$ is also proportional to the weakening of the Hadley cell. The correlation coefficient between $\Delta \chi$ and $\Delta \overline{[\mathrm{OLR}]}$ reaches $-0.61(-0.60)$ without (with) LASG, which is significant at the $99 \%$ confidence level. These results suggest that the weakening of the Hadley cells in other AGCMs is mainly balanced with the decrease of condensation heating and the increase of radiative cooling due to the suppression of deep convection, which reinforces our results shown in the previous section.

When the zonally asymmetric SST anomaly is added, the direction of change in the tropical climate is consistent across all AGCMs, this strongly suggests that the existence of a dynamics which dominates the direction. The significant correlation indicates that the positive feedback between largescale circulation and deep convection dominates the direction of change in the Hadley cell strength.

\footnotetext{
${ }^{4}$ The streamfunction response in each model is averaged over $0^{\circ}-20^{\circ} \mathrm{N}$ and $1000-150 \mathrm{hPa}$.
} 

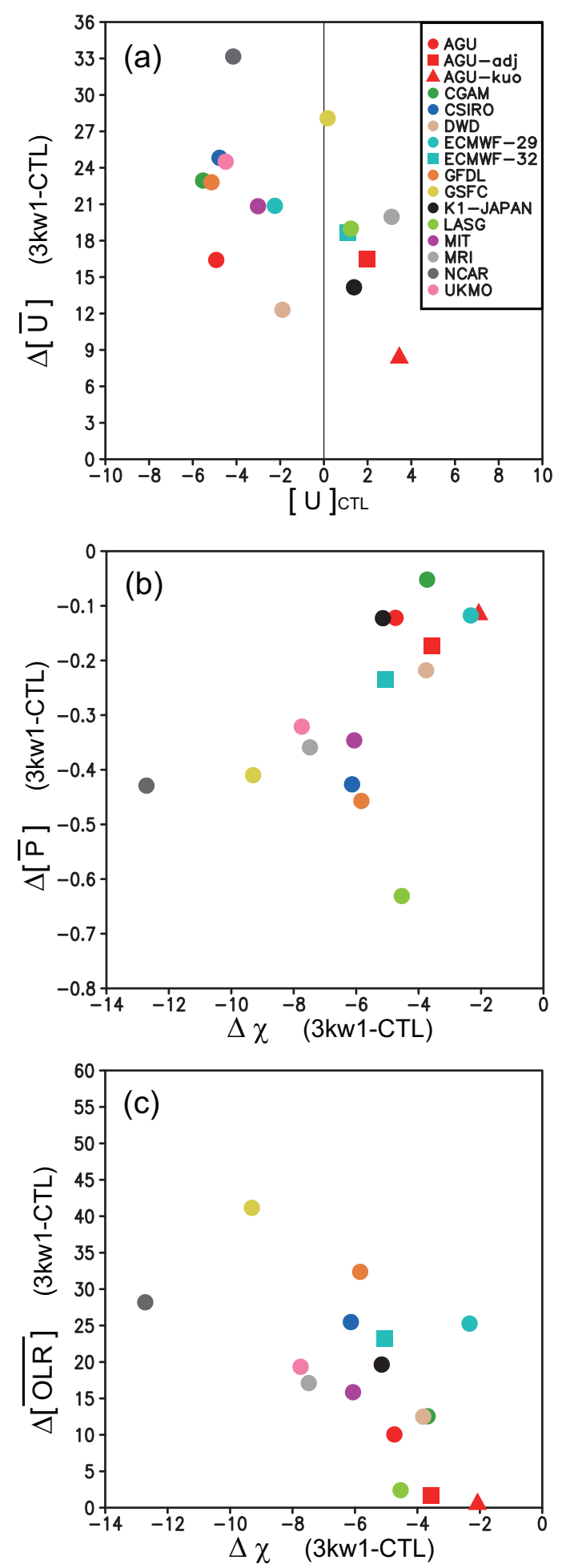

Fig. 15. The scatter diagram for (a) $\overline{[u]}_{\mathrm{ctl}}$ against $\Delta[u]$ (unit is $\mathrm{m} \mathrm{s}^{-1}$ ), (b) $\Delta \chi$ (unit is $1 \times 10^{3} \mathrm{~m} \mathrm{~s}^{-1}$ $\mathrm{Pa}$ ) against $\Delta \overline{[P]}$ (unit is $1 \times 10^{-4} \mathrm{~kg} \mathrm{~m}^{-2} \mathrm{~s}^{-1}$ ), and (c) $\Delta \chi$ against $\Delta \overline{[O L R]}$ (unit is $\mathrm{W} \mathrm{m}^{-2}$ ).

\section{Summary and discussion}

\subsection{Summary}

We examined the responses of the equatorial zonal wind and the Hadley circulation to an equatorial zonal wavenumber one SST anomaly $T_{s}{ }^{*}$ with varying magnitude controlled by $\alpha$ in an aquaplanet AGCM. The time- and zonal-mean equatorial zonal wind in the upper troposphere is a weak westerly in the control experiment driven by a zonally uniform SST, and is strengthened and extended into mid-troposphere as $\alpha$ increases. The equatorial momentum budget analysis illustrates that superrotation in the control experiment is maintained by the momentum flux convergence associated with transient eddies forced mainly by SCC, but the zonal acceleration associated with steady eddies is dominant for large zonal asymmetry in SST. This is caused by the suppression of the SCC over cold $T_{s}^{*}$ and the inhibition of poleward propagating transient waves by a stationary eddy over warm $T_{s}^{*}$.

The Hadley cell is weakened as $\alpha$ increases. We found that the weakening of the cell is balanced by the decrease in mean equatorial diabatic heating, which reflects a nonlinear relationship between tropical precipitation and SST. Namely, the precipitation decrease over cold $T_{s}^{*}$ is larger than the precipitation increase over warm $T_{s}^{*}$. The nonlinear relationship occurs due to an interaction between large-scale atmospheric circulation and deep convection. The SST anomaly excites the Walker circulation, which induces the subsidence anomaly over cold $T_{s}{ }^{*}$. Because the stabilization of lower troposphere and the decrease of relative humidity are induced by the subsidence anomaly over cold $T_{s}^{*}$, deep convection such as a SCC is effectively suppressed over there, and the zonally asymmetric response of precipitation and diabatic heating occurs (dynamic and thermodynamic change). The subsidence anomaly is also strengthened to balance the decrease of diabatic heating, and the zonally asymmetric precipitation response is further reinforced. Therefore, this positive feedback between the large-scale circulation and the transient convective disturbances determines the nonlinear relationship between precipitation and SST and controls the strength of the Hadley cell. In terms of the energetics of the tropical circulation, the Hadley cell has to be weakened to compensate for the lack of energy supply caused by an increase of tropical radiative cooling due to the effective suppression of deep convection over cold $T_{s}^{*}$.

Intercomparison of the 16 aquaplanet experiments using identical SST distribution demonstrates that the superrotation and weakening of the Hadley cell 
occur in all of the AGCMs when $T_{s}{ }^{*}$ is added to the zonally uniform SST. A large diversity with respect to the magnitude of changes suggests that the sensitivity of the tropical circulation change to $T_{s}^{*}$ greatly depends on the physical parameterization schemes in these AGCMs. Indeed the extent to which the Hadley cell is weakened roughly proportional to the decrease of tropical convective precipitation. This significant relationship indicates that the cell strength is controlled by the interaction between large-scale circulation and deep convection.

\subsection{Discussion}

The results of the momentum budget analysis for the time- and zonal-mean zonal wind are consistent with those of the previous studies using the dry idealized AGCM (KH05) except for the case of the CTL experiment. In our AGCM, the equatorial mean zonal wind for CTL is a weak westerly in the upper troposphere and is accelerated by the momentum flux convergence associated with the transient eddies forced mainly by SCC. This process cannot be represented in a dry model in which a heat source is imposed.

The difference in Hadley cell response between moist and dry models may be more crucial. The Hadley cell in the dry model does not change by the prescribed $[\bar{Q}]$ but by $Q^{*}$ (Fig. 1a). Thus, it is anticipated that the following thermodynamic balance relation approximately applies at the equator because $\Delta[\bar{Q}]=0$.

$$
\overline{\left[S_{p}\right]} \Delta \overline{[\omega]} \simeq-\overline{[\omega]} \Delta \overline{\left[S_{p}\right]}+\Delta \overline{[E]},
$$

where $\overline{[E]}$ represents eddy terms (see Eq. A3). In this case, the Hadley cell can change through $\Delta \overline{\left[S_{p}\right]}$ and $\Delta \overline{[E]}$. Because $\overline{\left[S_{p}\right]}$ is positive and $\overline{[\omega]}$ is negative in the middle to upper troposphere at the equator, by assuming that $\Delta \overline{[E]}$ is sufficiently small, we find that the weakening of the Hadley cell is balanced with positive $\Delta \overline{\left[S_{p}\right]}$. In this case, the weakening of the Hadley cell in the dry model may be accomplished by the warming in middle to upper troposphere.

While prescribing SST is useful for exactly controlling the temperatures, the surface not having ocean can respond to a nonphysical energy forcing. For instance, when SSTs are prescribed, the cloud decrease does not warm the surface via increased insolation, and evaporation anomalies are not associated with the warming of the surface. This kind of feedback that acts to inhibit unilateral change is not operated in AGCMs. Hence, the change in mean circulation shown in this study may be somewhat extreme. However, we believe that the interaction between large-scale circulation and deep convection shown in this study is also important for the formation of tropical climate in nature and coupled models.

Recently, using Tropical Rainfall Measuring Mission (TRMM) satellite data, Takayabu et al. (2010) showed that large-scale subsidence effectively suppresses deep convection, and they pointed out that the entrainment of mid to lower tropospheric dry air accomplished by the large-scale subsidence is the major factor for suppressing deep convection. Furthermore, Hirota et al. (2011) investigated the reproducibility of tropical precipitation in climate models participating in the Coupled Model Intercomparison Project phase 3 (CMIP3): They showed that low-score models overestimate (underestimate) precipitation over largescale subsidence (ascending) regions and suggested that suppression of deep convection due to the entrainment of dry air associated with subsidence is weak in low-score models. The behaviors of deep convection may be fed back to the formation of large-scale circulation itself. Therefore, these studies strongly suggest that the interaction between largescale circulation and deep convection is essential to form a tropical climate.

The relationship between the zonal asymmetry of SST and the strength of the Hadley cell may be essential not only for climate but also for interannual variability. Oort and Yienger (1996) showed using observational data that the Hadley cell is strengthened (weakened) during El Niño (La Niña). This is consistent with our results that the weak (strong) zonal asymmetry of SST invites the strong (weak) Hadley circulation. They have not fully explained its mechanisms, but the above similarity suggests the importance of the modulation of deep convection by the large-scale circulation associated with the change in Walker circulation to determining the Hadley cell variability.

\section{Acknowledgments}

We thanks to the participants in APE who conducted experiments. We also acknowledge two anonymous reviewers for their extremely helpful comments and suggestions for improving the manuscript. We also thank M. Yoshimori, M. Satoh, Y. Takayabu, M. Inatsu, and N. Hirota for beneficial discussions and comments. This work is supported by the Japanese Ministry of Education, Culture, Sports, Science, and Technology, through the Innovative Program of Climate Change Projection for the 21st Century. 


\section{Appendix A \\ Linear diagnostic equation for the meridional streamfunction}

The linear diagnostic equation for the meridional streamfunction is expressed below (Holton 2004). By applying the quasi-geostrophic scaling, time and zonal averaged zonal momentum, meridional momentum, thermodynamic, and hydrostatic balance equation in the pressure coordinate system can be written as follows:

$$
\begin{aligned}
& \frac{\partial \overline{[u]}}{\partial t}-f \overline{[v]}=\alpha_{x}, \\
& f \overline{[u]}+\frac{1}{a} \frac{\partial \overline{[\Phi]}}{\partial \varphi}=0, \\
& \frac{\partial \overline{[T]}}{\partial t}-\overline{\left[S_{p}\right]} \overline{[\omega]}=\alpha_{T}, \\
& \frac{\partial \overline{[\Phi]}}{\partial p}=-\frac{R \overline{[T]}}{p},
\end{aligned}
$$

where

$$
\begin{aligned}
& \overline{\left[S_{p}\right]}=\frac{\kappa}{p} \overline{[T]}-\frac{\partial \overline{[T]}}{\partial p}, \\
& \alpha_{x}=\overline{\left[F_{x}\right]}-\frac{1}{a \cos ^{2} \varphi} \frac{\partial}{\partial \varphi}\left(\left[\bar{u}^{*} \bar{v}^{*}\right] \cos ^{2} \varphi\right) \\
& -\frac{\partial}{\partial p}\left[\bar{u}^{*} \bar{\omega}^{*}\right]-\frac{1}{a \cos ^{2} \varphi} \frac{\partial}{\partial \varphi}\left(\overline{\left[u^{\prime} v^{\prime}\right]} \cos ^{2} \varphi\right) \\
& -\frac{\partial}{\partial p} \overline{\left[u^{\prime} \omega^{\prime}\right]} \\
& \alpha_{T}=\overline{\left[F_{T}\right]}+\frac{\overline{[J]}}{C_{p}}-\frac{1}{a \cos \varphi} \frac{\partial}{\partial \varphi}\left(\left[\bar{v}^{*} \bar{T}^{*}\right] \cos \varphi\right) \\
& -\frac{\partial}{\partial p}\left[\bar{\omega}^{*} \bar{T}^{*}\right]+\frac{\kappa}{p}\left[\bar{\omega}^{*} \bar{T}^{*}\right] \\
& -\frac{1}{a \cos \varphi} \frac{\partial}{\partial \varphi}\left(\overline{\left[v^{\prime} T^{\prime}\right]} \cos \varphi\right) \\
& -\frac{\partial}{\partial p} \overline{\left[\omega^{\prime} T^{\prime}\right]}+\frac{\kappa}{p} \overline{\left[\omega^{\prime} T^{\prime}\right]} \text {. }
\end{aligned}
$$

Here $u$ is the horizontal wind, $v$ the meridional wind, $\omega$ the vertical pressure velocity, $f$ the Coriolis parameter, $\Phi$ the geopotential, $T$ the temperature, $a$ the earth radius, $F_{x}$ and $F_{T}$ the frictional dissipation, $J$ the rate of diabatic heating per unit mass, $S_{p}$ the static stability, $R$ the gas constant, $C_{p}$ the heat capacity at constant pressure, and $\kappa=R / C_{p}$. For a variable $A, \bar{A}$ and $A^{\prime}$ denote time mean and deviation from the time mean, respec- tively, and $[A]$ and $A^{*}$ denote zonal-mean and deviation from the zonal-mean, respectively. The meridional streamfunction $\chi$ is defined as follows:

$$
\begin{aligned}
& \overline{[v]}=\frac{1}{\cos \varphi} \frac{\partial \chi}{\partial p}, \\
& \overline{[\omega]}=-\frac{1}{a \cos \varphi} \frac{\partial \chi}{\partial \varphi} .
\end{aligned}
$$

We differentiate (A2) with respect to time and substitute (A1); similarly, we differentiate (A4) with respect to time and substitute (A3). Both results are added to eliminate the time derivatives, and then using (A8) and (A9), the following linear diagnostic elliptic equation is obtained:

$$
\begin{aligned}
& f^{2} \frac{\partial^{2} \chi}{\partial p^{2}}+\frac{R}{a^{2} p}\left\{\left(\frac{\partial \overline{\left[S_{p}\right]}}{\partial \varphi}+\overline{\left[S_{p}\right]} \tan \varphi\right) \frac{\partial \chi}{\partial \varphi}\right. \\
& \left.\quad+\overline{\left[S_{p}\right]} \frac{\partial^{2} \chi}{\partial \varphi^{2}}\right\} \\
& \quad=-f \cos \varphi \frac{\partial \alpha_{x}}{\partial p}+\frac{R}{a p} \cos \varphi \frac{\partial \alpha_{T}}{\partial \varphi} .
\end{aligned}
$$

\section{References}

Arakawa, A., and W. H. Schubert, 1974: Interaction of a cumulus cloud ensemble with the large-scale environment, Part I. J. Atmos. Sci., 31, 674-701.

Battisti, D., and D. Ovens, 1995: The dependence of the low-level equatorial easterly jet on Hadley and Walker circulations. J. Atmos. Sci., 52, 3911-3931.

Blackburn, M., D. L. Williamson, K. Nakajima, W. Ohfuchi, Y. O. Takahashi, Y.-Y. Hayashi, H. Nakamura, M. Ishiwatari, J. McGregor, H. Borth, V. Wirth, H. Frank, P. Bechtold, N. P. Wedi, H. Tomita, M. Satoh, M. Zhao, I. M. Held, M. J. Suarez, M.-I. Lee, M. Watanabe, M. Kimoto, Y. Liu, Z. Wang, A. Molod, K. Rajendran, A. Kitoh, and R. Stratton, 2013: The Aqua-Planet Experiment (APE): CONTROL SST simulation. J. Meteor. Soc. Japan, 91A, 17-56, doi:10.2151/jmsj.2013-A02.

Bony, S., J.-L. Dufresne, H. Le Treut, J. J. Morcrette, and C. Senior, 2004: On dynamic and thermodynamic components of cloud changes. Climate Dyn., 22, 71-86.

Emori, S., and S. J. Brown, 2005: Dynamic and thermodynamic changes in mean and extreme precipitation under changed climate. Geophys. Res. Lett., 32, L17706, doi:10.1029/2005GL023272.

Frierson, D. M. W., 2007: The dynamics of idealized convection schemes and their effect on the zonally averaged tropical circulation. J. Atmos. Sci., 64, 1959-1976.

Gadgil, S., N. V. Joshi, and P. V. Joseph, 1984: Ocean-atmosphere coupling over monsoon regions. Nature, 312, 141-143.

Gill, A. E., 1980: Some simple solutions for heat-induced 
tropical circulation. Quart. J. Roy. Meteor. Soc., 106, 447-462.

Graham, N. E., and T. P. Barnett, 1987: Sea surface temperature, surface wind divergence, and convection over tropical oceans. Science, 238, 657-659.

Hasumi, H., and S. Emori, 2004: K-1 coupled model (MIROC) description., K-1 Tech. Rep., 1, 1-34. Center for Climate System Research, University of Tokyo

Hayashi, Y.-Y., and A. Sumi, 1986: The 30-40 day oscillations simulated in an "aqua planet" model. J. Meteor. Soc. Japan, 64, 451-467.

Held, I. M., and A. Y. Hou, 1980: Nonlinear axially symmetric circulations in a nearly inviscid atmosphere. J. Atmos. Sci., 37, 515-533.

Hide, R., 1969: Dynamics of the atmospheres of the major planets with an appendix on the viscous boundary layer at the rigid bounding surface of an electrically-conducting rotating fluid in the presence of a magnetic field. J. Atmos. Sci., 26, 841-853.

Hirota, N., Y. N. Takayabu, M. Watanabe, and M. Kimoto, 2011: Precipitation reproducibility over tropical oceans and its relationship to the double ITCZ problem in CMIP3 and MIROC5 climate models. J. Climate, 24, 4859-4873.

Holton, J., 2004: An introduction to dynamic meteorology. Academic press, $535 \mathrm{pp}$.

Hoskins, B., R. Neale, M. Rodwell, and G. Yang, 1999: Aspects of the large-scale tropical atmospheric circulation. Tellus, 51A-B, 33-44.

Inatsu, M., H. Mukougawa, and S.-P. Xie, 2002: Stationary eddy response to surface boundary forcing: Idealized GCM experiments. J. Atmos. Sci., 59, 1898-1915.

Kimoto, M., 2005: Simulated change of the east Asian circulation under global warming scenario. Geophys. Res. Lett., 32, L16701, doi:10.1029/2005GL023383.

Kraucunas, I., and D. L. Hartmann, 2005: Equatorial superrotation and the factors controlling the zonal-mean zonal winds in the tropical upper troposphere. J. Atmos. Sci., 62, 371-389.

Lee, S., 1999: Why are the climatological zonal winds easterly in the equatorial upper troposphere?. J. Atmos. Sci., 56, 1353-1363.

Lin, J.-L., G. N. Kiladis, B. E. Mapes, K. M. Weickmann, K. R. Sperber, W. Lin, M. C. Wheeler, S. D. Schubert, A. Del Genio, L. J. Donner, S. Emori, J.-F. Guermy, F. Hourdin, P. J. Rasch, E. Roeckner, and J. F. Scinocca, 2006: Tropical intraseasonal variability in 14 IPCC AR4 climate models. Part I: Convective signals. J. Climate, 19, 2665-2690.

Matsuno, T., 1966: Quasi-geostrophic motions in the equatorial area. J. Meteor. Soc. Japan, 44, 25-42.

Mochizuki, T., M. Ishii, M. Kimoto, Y. Chikamoto, M. Watanabe, T. Nozawa, T. T. Sakamoto, H. Shiogama, T. Awaji, N. Sugiura, T. Toyoda, S. Yasunaka, H. Tatebe, and M. Mori, 2010: Pacific decadal oscillation hindcasts relevant to near-term climate prediction. Proc.
Natl. Acad. Sci. U.S.A., 107, 1833-1837, doi:10.1073/ pnas.0906531107.

Nakazawa, T., 1988: Tropical super clusters within intraseasonal variations over the western Pacific. J. Meteor. Soc. Japan, 66, 823-839.

Nasuno, T., 2008: Equatorial mean zonal wind in a global nonhydrostatic aquaplanet experiment. J. Meteor. Soc. Japan, 86A, 219-236.

Neale, R. B., and B. J. Hoskins, 2001a: A standard test for AGCMs including their physical parametrizations: I: The proposal. Atmos. Sci. Lett., 1, 101-107, doi:10.1006/asle.2000.0019.

Neale, R. B., and B. J. Hoskins, 2001b: A standard test for AGCMs including their physical parametrizations. II: Results for the Met Office model. Atmos. Sci. Lett., 1, 108-114, doi:10.1006/asle.2000.0020.

Nozawa, T., T. Nagashima, H. Shiogama, and S. A. Crooks, 2005: Detecting natural influence on surface air temperature change in the early twentieth century. Geophys. Res. Lett., 32, L20719, doi:10.1029/2005GL023540.

Numaguti, A., 1993: Dynamics and energy balance of the Hadly circulation and the tropical precipitation zones: Significance of the distribution of evaporation. $J$. Atmos. Sci., 50, 1874-1887.

Oort, A. H., and J. J. Yienger, 1996: Observed interannual variability in the Hadley circulation and its connection to ENSO. J. Climate, 9, 2751-2767.

Pan, D., and D. A. Randall, 1998: A cumulus parametrization with a prognostic closure. Quart. J. Roy. Meteor. Soc., 124, 949-981.

Pierrehumbert, R. T., 1995: Thermostats, radiator fins, and the local runaway greenhouse. J. Atmos. Sci., 52, 1784-1806.

Rayner, N. A., D. E. Parker, E. B. Horton, C. K. Folland, L. V. Alexander, D. P. Rowell, E. C. Kent, and A. Kaplan, 2003: Global analyses of sea surface temperature, sea ice, and night marine air temperature since the late nineteenth century. J. Geophys. Res., 108, 4407, doi:10.1029/2002JD002670.

Saravanan, R., 1993: Equatorial superrotation and maintenance of the general circulation in two-level models. J. Atmos. Sci., 50, 1211-1227.

Satoh, M., 1994: Hadley circulations in radiative-convective equilibrium in an axially symmetric atmosphere. $J$. Atmos. Sci., 51, 1947-1968.

Suarez, M. J., and D. G. Duffy, 1992: Terrestrial superrotation: A bifurcation of the general circulation. J. Atmos. Sci., 49, 1541-1554.

Takayabu, Y. N., S. Shige, W.-K. Tao, and N. Hirota, 2010: Shallow and deep latent heating modes over tropical oceans observed with TRMM PR spectral latent heating data. J. Climate, 23, 2030-2046.

Uppala, S. M., and Coauthors, 2005: The ERA-40 re-analysis. Quart. J. Roy. Meteor. Soc., 131, 2961-3012.

Watanabe, M., 2008: Two regimes of the equatorial warm 
pool. Part II: Hybrid coupled GCM experiments. J. Climate, 21, 3545-3560.

Williamson, D. L., M. Blackburn, B. Hoskins, K. Nakajima, W. Ohfuchi, Y. O. Takahashi, Y.-Y. Hayashi, H. Nakamura, M. Ishiwatari, J. McGregor, H. Borth, V. Wirth, H. Frank, P. Bechtold, N. P. Wedi, H. Tomita, M. Satoh, M. Zhao, I. M. Held, M. J. Suarez, M.-I. Lee, M. Watanabe, M. Kimoto, Y. Liu, Z. Wang, A. Molod, K. Rajendran, A. Kitoh, and R. Stratton, 2012: The APE Atlas. NCAR tech. Note NCAR/TN-484+STR, National Center for Atmospheric Research, Boulder, Colorado, xxii+508 pp. [Available at http://nldr.library.ucar.edu/repository/
collections/TECH-NOTE-000-000-000-865.]

Williamson, D. L., M. Blackburn, K. Nakajima, W. Ohfuchi, Y. O. Takahashi, Y.-Y. Hayashi, H. Nakamura, M. Ishiwatari, J. McGregor, H. Borth, V. Wirth, H. Frank, P. Bechtold, N. P. Wedi, H. Tomita, M. Satoh, M. Zhao, I. M. Held, M. J. Suarez, M.-I. Lee, M. Watanabe, M. Kimoto, Y. Liu, Z.Wang, A. Molod, K. Rajendran, A. Kitoh, and R. Stratton, 2013: The Aqua-Planet Experiment (APE): Response to changed meridional SST profile. J. Meteor. Soc. Japan, 91A, 57-89, doi:10.2151/jmsj.2013-A03. 